\title{
Reliable and Accurate Solution to the Induced Fit Docking Problem for Protein-Ligand Binding
}

Edward B. Miller, Robert B. Murphy, Daniel Sindhikara, Kenneth W. Borrelli, Matthew J. Grisewood, Fabio Ranalli, Steven L. Dixon, Steven Jerome, Nicholas A. Boyles, Tyler Day, Phani Ghanakota, Sayan Mondal, Salma B. Rafi, Dawn M. Troast, Robert Abel, Richard A.

Friesner 
I Detailed Description of the IFD-MD Workflow

I.A Initial Docking Stage

I.A.1 Statement of The Problem

We consider two protein-ligand complexes of a receptor $\mathrm{R}$, which we shall designate as complexes $\mathrm{A}$ and $\mathrm{B}$. The ligands in these complexes will be referred to as ligand $A\left(L_{A}\right)$ and ligand $B\left(L_{B}\right)$, respectively. The goal is to dock $L_{A}$ into receptor $\mathrm{B}\left(\mathrm{R}_{\mathrm{B}}\right)$ and obtain an accurate pose and receptor structure (in a retrospective test case, presumably that given by complex A).

For ease of discussion, the ligand in $\mathrm{R}_{\mathrm{B}}$, Ligand $\mathrm{B}$, formally called $\mathrm{L}_{\mathrm{B}}$, shall be referred to going forward as the template ligand. And the ligand whose structure is sought within $\mathrm{R}_{\mathrm{B}}$, shall be referred to as the target ligand. This nomenclature arises naturally in the context of the overall workflow described below.

We consider cases where rigid receptor docking of the target ligand into $\mathrm{R}_{\mathrm{B}}$ results in a poor structure, presumably due to one or more steric clashes. In rigid receptor docking, neither the side chains nor the backbone of $\mathrm{R}_{B}$ is allowed to move. Hence, the first challenge in any efficient IFD protocol is to generate an initial pose for the target ligand in $R_{B}$ that is reasonably close to the experimental result, without expending inordinate amounts of computer time (e.g. as would be the case using an explicit solvent MD simulation for this initial phase of the calculation). As was noted above, we will focus in the present paper primarily on cases where side chain motion is sufficient to enable such a structure to be produced (small backbone motions can be introduced later via MD and/or minimization).

In our previous work ${ }^{1}$, the principal strategy was to mutate a subset of side chains to alanine. Here, we augment this strategy by also creating receptor conformations in which one or more side chains are moved into a different rotamer state. A large ensemble of receptor conformations is developed in which alanine mutations and new rotamer states can both be present. A substantial enlargement of the ensemble size, combined with the option of rotamer state changes and an improved approach to identifying key side chains to be moved (discussed below), results in the generation of at least one "close enough" pose across a wide diversity of IFD test cases. Because rigid receptor docking into each conformation via GlideSP is extremely fast (on the order of a few CPU seconds), the use of thousands or even tens of thousands of conformations is unproblematic from the point of view of overall computational time for the full workflow. The problem of extracting a small subset of candidate poses for more extensive computational exploration from the huge number of alternatives generated by this protocol is outlined in the next few sections.

\section{I.A.2 Use of Pharmacophore-Based Docking to Identify Side Chains to Mutate or Move Into Different Rotamer States}

Pharmacophore based alignments have been used for many years to identify ligands which have the capacity to bind analogously to known active compounds. A given pharmacophore superposition is often wrong or incomplete, particularly when the ligand being aligned is not very chemically similar to the known active template. However, if we consider many different possible target ligand conformations and alignments, one of them often captures key features of the correct binding mode, even if its alignment score would ordinarily be insufficient to select it as such.

In the present case, the template ligand is situated in its crystallographic binding mode in the receptor, and standard Phase based pharmacophore alignment is carried out using many different conformations of the target ligand. These alignments are then examined in the context of the binding site to identify possible steric clashes with side chains (note that the same approach can readily be deployed to suggest possible backbone clashes, an avenue we plan to explore in generalizing IFDMD to cases where significant backbone motion is required). The ligand conformations are ranked based on their pharmacophore overlap. The list of conformations is traversed in order of descending overlap, each time ultimately yielding a potentially new receptor conformation via the use of Prime to resolve any side chain clashes, discussed in detail below.

Crucially, the pharmacophore-docking of the target ligand onto the template ligand is done ignoring both clashes and favorable interactions of the target ligand with the receptor. This avoids the need to first alter the receptor before the initial docking of the ligand, a formidable challenge which was part of our prior IFD algorithm ${ }^{1}$, and many others ${ }^{2-4}$. The fact that our new IFD-MD algorithm here avoids this necessity is one of its distinguishing characteristics.

\section{I.A.3 Prime Side Chain Refinement Generates Alternative Receptor Conformations}

For a pharmacophore-docked target ligand conformation, a list of side chains is assembled which have one or more atoms having a van der Waals clash with the target ligand. The side chain clash is resolved in the presence of the docked ligand using Prime's binding site refinement algorithm ${ }^{5-8}$. Briefly, Prime's side chain algorithm uses a $10^{\circ}$-resolution side chain 
rotamer library to sample side chain conformations. The positions of the side chains selected for refinement are randomized and then iteratively optimized until no clashes are present and the results are self-consistent. Once the side chain rotamers have converged, the side chain atoms are minimized with the OPLS3e forcefield and the VSGB2 ${ }^{8}$ solvent model.

It is crucial that the side chain clash be resolved in the presence of the pharmacophore docked ligand so as to allow possible favorable interactions with the ligand, even if the ligand conformation is itself only crudely docked. Furthermore, the simple existence of the ligand atoms will ensure the side chain sampling and scoring focuses on rotamers which accommodate a docked ligand rather than occlude the binding site. This is a significant departure from our prior IFD algorithm which selected side chains to truncate based on solvent exposed surface area or B-factors, without any putative ligand present in the binding site $^{1}$. It is still possible that the pharmacophore docking may place the ligand so crudely as to prevent any refinement of a side chain clash. To provide robustness, mutated receptors are also generated with the clashing side chain truncated to alanine. Mutations and side chain conformation changes are not both done within a single generated receptor structure. For any alternative receptor that is generated, the target ligand is deleted leaving a new apo-receptor structure available for docking with GlideSP.

Care is taken to remove redundant receptor conformations by measuring side chain RMSDs against previously generated conformations. Once a sufficient number of alternative receptor conformations are produced, the search through the pharmacophore docked ligand conformations is terminated. Sufficiency in receptor conformations is defined by criteria for both the total number of novel receptor conformations, and the number of alternative conformations for a subset of side chains.

We have found that allowing up to a total of six side chains to be simultaneously sampled, or eight side chains to be mutated, is sufficient to address all of the test cases we have studied to date (including a number where there were quite drastic differences in structure between the target and the template). Although fewer side chains are permitted to be conformationally sampled than can be mutated, the vast majority of alternative receptor structures produced are those with side chain conformation changes. For any single side chain there may be multiple clash-free positions and the combinatorics of multiple side chains positions is what dominates.

\section{I.A.4 GlideSP Docking Generates Initial Set of Candidate Ligand-Receptor Complexes}

The combination of pharmacophore docking combined with Prime side chain sampling yields up to 1000 unique receptor conformations. The target ligand is then rigid-receptor docked into each of these unique receptor conformations using GlideSP. Provided then that a favorable re-arrangement of the side chains has been performed, rigid-receptor docking should be successful in sampling and ranking a native-like pose with at least one receptor conformation.

However, docking into up to 1000 unique receptor conformations produces an enormous number of possible ligandreceptor complex conformations which must be paired down in some manner. Although the rigid-receptor docking score may be suitable for ranking ligand conformations docked into a single receptor conformation, this score, if used here as an exclusive metric, would fail to take in to account the relative energetics of the receptor conformations. This means that using the docking score to rank predicted complexes across receptor conformations is far too inaccurate.

To proceed further, the ligand conformations are clustered to remove near duplicates, and then the top 1000 ligand conformations, across all rigid-receptor jobs are retained, as ranked by the GlideSP score. These ligand conformations are then placed back in to the original template receptor. This naturally re-introduces ligand-receptor atom clashes but provides a new opportunity to refine the side chains around docked ligand conformation. Here, however, the ligand conformation was generated using GlideSP rather than with the cruder pharmacophore-based methods of Phase which deliberately did not explicitly consider receptor-ligand interactions.

I.B Two Iterations of Prime Side Chain Sampling and Minimization, Followed by Redocking with GlideSP, Generates a Short List of Candidate Poses for Further Study

With the ligand having been re-docked using GlideSP rather than using Phase's pharmacophore-based docking, Prime side chain optimization is performed followed by minimization of all surrounding side chains and the ligand. Here, Glide's docking of the ligand was done fully taking the receptor into account. Therefore, severe ligand-receptor clashes are not expected and Prime's side chain refinement is unlikely to result in large side chain motions.

Because it will become necessary to compare the scores of all the conformations of the ligand-receptor complex emerging from the initial candidates, regardless of origin, it is important that the number of degrees of freedom included in side chain sampling and minimization are kept constant across all intermediate optimization steps. To satisfy this requirement, the set 
of side chains refined is the union, across all receptors, of side chains within $5 \AA$ of the ligand. Due to the diversity of generated complexes, this list can become rather large, comprising between 50 and 100 side chains.

Upon convergence of the side chain rotamers, we permit minimization of both the ligand and all atoms on each residue, including both backbone and side chain atoms. We have found this permits slight relaxations of backbone clashes in the presence of the ligand. Although not sufficient to reliably handle large loop motions, this minimization step does remove the algorithm from being hypersensitive to small backbone adjustments.

At this point, one has both a GlideSP docking score and a Prime continuum solvation energy for each ligand-receptor complex. However, the GlideSP score refers to the ligand prior to side chain optimization and minimization and therefore is potentially no longer relevant. For example, additional ligand-receptor interactions may have formed during side chain sampling. In principal, iterative ligand resampling with GlideSP followed by receptor sampling with Prime can be done until a defined convergence is reached. In practice we have found it sufficient to repeat GlideSP docking and Prime refinement only once more. As we discuss further below, there are severe limitations in ranking predicted complexes using just the GlideSP docking score and the Prime continuum solvation energy. Therefore, further Glide/Prime iterations are not expected to provide improved ranking.

II Preliminary Scoring and Clustering Using the Prime Energy and GlideSP Scores

From our previous efforts to rank IFD poses ${ }^{1}$, we have seen that a linear combination of the Prime continuum solvation energy, GlideSP docking score, and GlideSP ECoul term is capable of ranking a native like pose within the top 10 (typically) and top 20 (almost always) poses, assuming that such a pose is generated by the Phase, Glide, and Prime (PGP) steps described above. Here we apply the same scoring function to select representative members for clustering.

Clustering is performed via ligand pairwise RMSD calculations. To avoid the full cost of $\mathrm{N}^{2} \mathrm{RMSD}$ calculations, first the RMSD of a yet-to-be-clustered pose is computed against representative cluster members. If the RMSD to the representative member is $2 \AA$ or less, then individual RMSD calculations are performed on each member of that cluster. The yet-to-beclustered pose will be added to an existing cluster if its RMSD is less than $1.5 \AA$ to any member of that cluster. There are typically well over 100 unique clusters produced.

As will be documented in detail below, the total PGP docking stage produces a suitable pose, ranked within the top 20 structures, in $99 \%$ of the cases across both the 258 training set cases, and 157 test set cases. Thus, as long as changes in the receptor backbone are not too great, the PGP stage of IFD-MD provides a robust approach to producing a tractable number of candidate poses for further processing.

III Rescoring the Top 20 candidate Poses via Short MD Simulations and a WScore Evaluation of the Final Group of MD poses

We recommend examining the top twenty poses in this next stage of IFD-MD; the computational cost is not terribly high, and a small but nontrivial improvement in robustness is obtained across both the training and test sets. For challenging problems such as homology modeling or when there are drastic differences between the target and template ligand, it may make sense to retain more poses for the metadynamics simulations, depending upon the score gap between the top scoring poses and the additional poses beyond the $20^{\text {th }}$ position.

Each of the top twenty poses emerging from the PGP ranking is subject to ten different 500 ps molecular dynamics simulations. The Schrodinger solvate pocket algorithm for Desmond, which utilizes grand canonical Monte Carlo (GCMC) to make sure that water molecules are properly placed into the complex, is essential in scoring a subset of cases (and also in preparing them for metadynamics).

The idea behind the MD runs is twofold. Firstly, the molecular dynamics simulations relax any close contacts or other significant problems that are present in the IFD structure, which has to date only employed minimization to move the backbone. Secondly, the WaterMap'-based desolvation scoring in WScore is subject to error if an imprecise receptor structure buries a ligand or protein polar/charged group. The short MD simulation will in most cases, if the structure is reasonably close to the native structure, move the protein backbone so as to enable solvation of the desolvated group in question. Imagine for example that a solvent exposed loop has an $\mathrm{NH}$ group pointing at a ligand hydrophobic moiety at a close distance, which is therefore desolvated. In the MD simulation, the loop may adopt a modified conformation in which the NH group is able to hydrogen bond to a water molecule. Figure S1 presents an example showing a picture of an original IFD pose (indicating the desolvated $\mathrm{NH}$ group) and the structure that results from a $500 \mathrm{ps}$ MD simulation, highlighting the modified backbone conformation of the loop, and the water molecule that now binds to the NH group. 

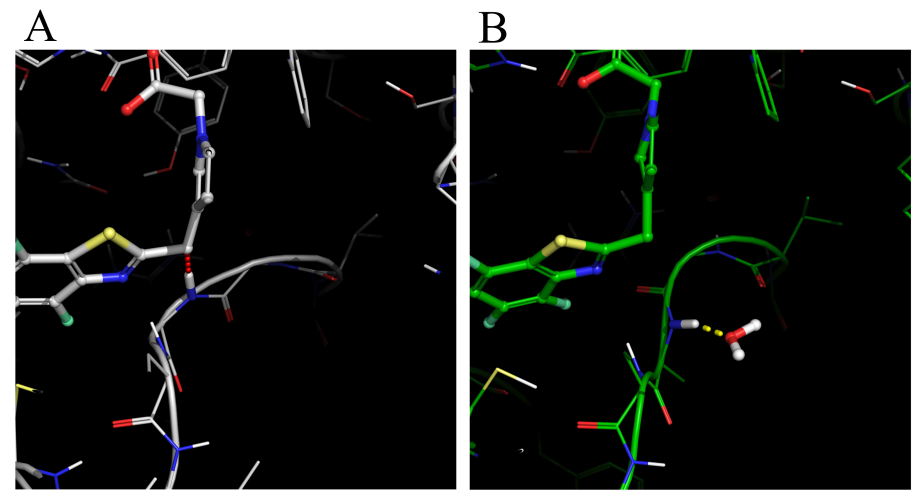

Figure S1. Motion of the protein backbone over the course of a 500 ps MD simulation. The backbone motion corrects for the desolvation of an NH group. (A) The position of the backbone in the original IFD pose, prior to any molecular dynamics. The red dotted line highlights the close contact the $\mathrm{NH}$ group is making to a linker $\mathrm{sp}^{3}$ carbon in the ligand. In this position, the $\mathrm{NH}$ group is desolvated. (B) The system after $500 \mathrm{ps}$ of MD simulation. Despite the short timescale of the simulation, the MD has corrected this unfavorable interaction by rotating the $\mathrm{NH}$ group roughly $90^{\circ}$ and allowing for the interaction with an explicit MD water, shown by the yellow dotted line.

We have discussed the WScore docking and scoring approach in a previous publication ${ }^{10}$. The WScore scoring function is an empirical model that assigns favorable terms for protein-ligand hydrophobic and van der Waals interactions, hydrogen bonding, and specific types of electrostatic interactions, while penalizing ligand strain energy and desolvation of ligand or protein polar or charged groups. A detailed discussion of the terms in WScore, fitting of the model to experimental data, and performance in rank ordering and enrichment tests, can be found in ref ${ }^{10}$.

As noted above, the use of a short MD simulation prior to WScore evaluation enables the initial IFD structure to be improved, and improper desolvation of polar and charged groups to be relieved. The deployment of multiple independent MD trials is also essential in avoiding spurious penalty assignments for native-like structures. The question of how many such trials are necessary and sufficient to provide robust performance, without inordinate computational cost, is an empirical one, which we have studied in detail in assessing results for our training and test sets. Ten trials appear to be sufficient for all of the cases we have examined to date. As we accumulate more data on a wider range of relevant IFD problems (including prospective use in projects), it will become apparent whether an upward revision of this number is needed.

The WScore evaluations obtained from the ten MD trials are used in three ways. Firstly, if every pose has a significant WScore penalty (desolvation or strain energy), the pose is assigned a significant penalty term (the value of which is optimized along with the remainder of the IFD-MD scoring function). Tight binding ligands in the PDB rarely if ever have such penalties, and certainly will not choose to adopt such a pose if there is a comparable but penalty free alternative. The Prime MMGB/SA model cannot accurately make such distinctions, which depend upon a few $\mathrm{kcal} / \mathrm{mol}$ of energy, without considerably better sampling (and might have trouble even then for desolvation events involving individual water molecules, which are common in protein-ligand binding). Secondly, a smaller, but still useful, penalty, is applied depending upon the number of penalty-free MD poses obtained. Thirdly, the best total WScore value from among the ten poses is employed directly as a term in the final scoring function (with a coefficient which is optimized based on training set results).

The 20 IFD poses then go on to the next phase of IFD-MD, metadynamics pose scoring.

\section{Metadynamics Pose Scoring}

Metadynamics pose scoring is carried out as described in ref. ${ }^{11}$. To briefly review the arguments in that paper, the goal is to assess the stability of each pose using explicit solvent simulation and the OPLS3e force field ${ }^{12}$, which has demonstrated its ability to accurately assess relative energetics in biomolecular simulations in a variety of contexts, including both protein conformational energetics and FEP calculations of relative protein-ligand binding affinities. A simple MD simulation will often fail at this task due to the metastability of incorrect binding poses. By applying an appropriately sized metadynamics force to the pose, using the ligand RMSD from the initial pose as the collective coordinate, the differential stability of the candidate poses is interrogated in a fashion more likely to yield a useful signal. There are poses which are metastable even under our current metadynamics protocol; however, these poses invariably have a flaw that can be detected by other components of the scoring function (e.g. a WScore desolvation penalty). In fact, our observations to date suggest that the various scoring terms are physically complementary. Data supporting this assertion will be presented in Section XII.C. 
One of the metrics evaluated over the course of our metadynamics simulation is the persistence of hydrogen bonds generated by the PGP stage. As discussed in ref. ${ }^{11}$ in addition to the positional stability of the ligand, the quality of the ligand pose can be inferred by the stability of hydrogen bonds created prior to the start of the simulation. The formation of hydrogen bonds during the PGP steps is done without any consideration of entropic costs. Therefore, the loss of hydrogens bonds over the course of the metadynamics simulation can provide an indication of the free energy gains upon binding (or lack thereof) due to particular hydrogen bonds. As a departure from ref. ${ }^{11}$, the measured hydrogen bond persistence is used in IFD-MD as a penalty term. This term augments the favorable energetics present in Prime's implicit solvent energy model and the GlideSP scoring function by acting as a correction.

\section{Penalty for Large Movement of Buried Residues}

In a number of cases, the PGP steps produce poses in which there are significant displacements of aromatic or charged residues in the protein core, which are necessary to accommodate the target ligand in the pose in question. Such displacements do occur in experimental observed induced fit binding, but are very infrequent, as there is a significant reorganization free energy cost. Consequentially, we have developed terms in the scoring function which penalize such large displacements. The values of each penalty term are optimized along with the other scoring function components. Cases where a displacement is required to achieve an accurate induced fit structure serve as a control; if the penalty is too large, the wrong pose will be selected in such cases. The objective is to tip the balance towards the less disruptive pose in cases where the scores are relatively close.

The benefits of the inclusion of this term are discussed in more detail in Section XII.C, with visualization of illustrative examples. However, it should be noted that there are a relatively small number of cases (15 out of 415) where it is relevant. More data may reveal useful design improvements in this type of term, including extension to other residue types.

\section{Composite Scoring Function for Ranking Poses}

The next step in IFD-MD is to rank the top 20 poses from the PGP stage using a composite scoring function constructed from the various terms described above.

In order to optimize the parameters of the scoring function, we first assign poses in all of the training set cases as acceptable or not acceptable. This is not a completely straightforward exercise. A pose with a sufficiently low RMSD is acceptable, but what should the cutoff be? The objective at this stage is not to produce the final pose, but rather to eliminate non-native basins of attraction from consideration.

After some experimentation, we have selected a ligand heavy-atom RMSD cutoff (to the native structure) of $2.5 \AA$ as signaling an acceptable pose. In most cases, the crystal structure is used as a comparison. In a few instances, poses are labeled as acceptable which have a solvent exposed tail region that is non-native, leading to a larger RMSD to the crystal structure, which does not collapse to a native like position in a 500 ps MD run, but displays a distribution of ligand poses that is very similar to an MD simulation starting from the native structure in a longer 100 ns run (see Section XII.C for an enumeration of these cases). We quantify this by comparing the 100 ns native MD trajectory against the predicted ligand pose. To distinguish a transient incidental similarity, we require that at least $25 \%$ of the native MD trajectory lie within 1.5 $\AA$ heavy atom ligand RMSD of the predicted pose. When this is observed, we consider the predicted pose to lie within the native molecular dynamic basin.

Once the acceptable poses are labelled, a total of 18 parameters are optimized to combine the score of the various terms into a composite score.

The functional form chosen is as follows:

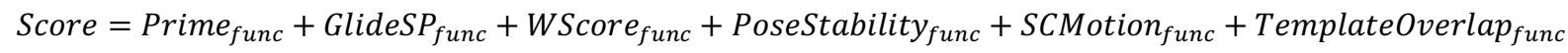

Equation 1. IFD-MD Scoring Function

Where each of the individual functions is as follows:

Prime $_{\text {func }}=w_{1} \Delta$ Prime $_{\text {Energy }}$

Equation 2. Prime Sub-Function 
where $\mathrm{w}_{1}$ refers to a weight for this term and is a fitted parameter. The $\Delta$ refers to the change in Prime Energy $_{\text {across the top }}$ 20 poses from the PGP stage, relative to the largest value. For all the following functions, the $\Delta$ refers to a similar change in any term across the 20 poses.

GlideSP $_{\text {func }}=w_{2} \Delta$ Glide Docking $_{3}+w_{3} \Delta$ Glide ECoul $_{\text {Cl }}$

Equation 3. Glide Sub-Function

where Glide Docking $_{\text {and Glide }}$ Eoul refers to the GlideSP docking and ECoul scores generated from the PGP stage.

$W_{\text {Score }}$ func $_{1}=w_{4}$ AllMDTrialsPenalized $+w_{5} \max \left(\right.$ Num $\left._{\text {WScorePenaltyMDTrials }}-5,0\right)+w_{6} \Delta$ WScore $_{\text {BestTrial }}+$

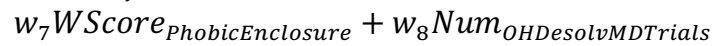

Equation 4. WScore Sub-Function

where AllMDTrialsPenalized refers to whether all the 500 ps MD trials contain at least one WScore penalty. Num $_{W S c o r e P e n a l t y M D T r i a l s}$ refers to the count of MD trials which have at least one WScore penalty. WScore BestTrial is the best WScore docking score seen across all the MD trials for a given pose. WScore PhobicEnclosure is the WScore phobic enclosure

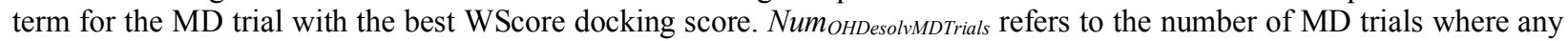
ligand hydroxyl group appears desolvated.

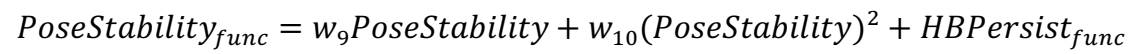

Equation 5. PoseStability Sub-Function

where PoseStability comes from the metadynamics pose scoring discussed above and in ref ${ }^{11}$. Qualitatively, PoseStability is computed from the RMSD of the ligand at the end of the metadynamics simulation versus its starting position. Stability of the pose is also inferred from the persistence of hydrogen bonds over the course of the metadynamics simulation. This is quantified by the HBPersist func $_{\text {. }}$

HBPersist $_{\text {func }}=5\left\{\begin{array}{cc}1 & \text { if } h b_{\text {persist }} \leq P_{1} \\ \frac{h b_{\text {persist }}}{P_{1}-P_{2}}+\frac{P_{2}}{P_{2}-P_{1}} & \text { if } P_{1}>h b_{\text {persist }}>P_{2} \\ 0 & \text { if } h b_{\text {persist }} \geq P_{2}\end{array}\right.$

Equation 6. Hydrogen Bond Persistence Sub-Function

where $h b_{\text {persist }}$ refers to the fractional persistence (ranging from zero to one) of all ligand-receptor hydrogen bonds over the course of the metadynamics simulation discussed above. This function here is a ramp function that changes from one to zero within $h b_{\text {persist }}$ values between $P_{l}$ and $P_{2}$. These two terms define the region where the function linearly changes and were treated as fitted parameters.

SCMotion $_{\text {func }}=\max \left(\sum S C_{P e n, P H E}+S C_{\text {Pen,MET }}+S C_{\text {Pen,General }}, M_{S C P e n, G e n e r a l}\right)$

Equation 7. Side-Chain Motion Sub-Function

where each of the $S C_{P e n}$ terms is

$S C_{\text {pen }}=\max \left(w_{11}\right.$ Num $\left.{ }_{\text {SCMotionMDTrials }}, M_{S C P e n}\right)$.

Equation 8. Individual Side-Chain Penalty 
Put in words, each penalty for the motion of a large buried residue is weighted and capped. For PHE and MET, a separate cap is applied, and the sum over all side chain penalties is itself capped. These caps are also treated as fitted parameters.

TemplateOverlap func $_{12}=w_{12} \frac{1}{\text { Tempoverlap }+\epsilon}$

Equation 9. Template Overlap Sub-Function

where TempOverlap measures the volume overlap between a candidate target ligand pose and the template ligand. It is normalized to lie between zero and one. The identification of novel binding sites is considered beyond the scope of IFDMD and so this term here penalizes poses which share little volume overlap with the template ligand. The precise value of epsilon is treated as a fitted parameter.

The optimization of the terms is done using a grid search with a cost function that weights the number of cases where an acceptable pose was ranked first, the number of cases where an acceptable pose was ranked second, and the score gap between the worst scoring acceptable pose and the best scoring unacceptable pose.

The grid search involves precomputing the value of each individual term at all particular weights within a set of discretized weight values. The cost function's output for a given set of grid points is then just a summation. In practice, the enormous dimensionality of an 18-parameter grid space prevents exhaustive exploration. Instead, terms which are expected to be highly coupled are optimized simultaneously while other terms are kept fixed. For example, the hydrogen bond persistence parameters and the prime energy function parameter are optimized together. The hydrogen bond persistence function is expected to correct for Prime's energy model favoring hydrogen bonds which happen to not persist over the course of an MD simulation. The value of all parameters is initially randomized on the grid and the entire optimization repeated with different initial values to find the lowest cost.

To remove obvious overfitting, we also perform leave one out (LOO) validation for each IFD case in the training set. The results of fitting and LOO validation are discussed in Section XII.C.

\section{Refinement of an IFD-MD Pose}

The full set of IFD and simulation data is available from an IFD-MD run, giving the user a more complete view of the properties of the structure that has been produced than would be available from the PGP prediction alone. However, we also want to generate our "best" single structure approximation for the complex. In many cases, the experimental crystal structure is a useful proxy for an underlying low temperature single structure, around which fluctuations occur at room temperature. The use of crystal structures in designing potent and selective ligands is well validated over a wide range of experimental projects. Thus, selecting a structure with a low RMSD to the crystal structure would be viewed as an IFD success. There are also cases where an unbiased MD simulation, starting from the crystal structure, converges to a different underlying structure at sufficiently long simulation times (100 ns in the present case). While this could be due to errors in the MD force field, in our view it is more likely that the alternative basin of attraction is at the very least competitive in free energy at room temperature. Hence, in evaluating the RMSD of the IFD-MD selected single structure (the method for choosing which is described below), we consider the RMSD to both the crystal structure and the tail end of the 100ns MD simulation starting from the crystal structure. The algorithm for comparing to the native MD simulation was described in the previous section.

The next question is how to select the best single structure approximation for each top scoring IFD pose. We have ample evidence, over a large number of test cases, that selecting the pose with the best WScore (BWS) from among the twenty final poses emerging from the ensemble of $500 \mathrm{ps}$ MD simulations yields a significant improvement in quality as compared to the initial, PGP generated pose. Firstly, in many cases the MD pose relaxes towards the crystal structure, exhibiting a substantial improvement in RMSD. Secondly, the MD simulations efficiently remove many if not most of the WScore penalties, for example buried polar or charged groups, or internal energy clashes. The MD refined pose is thus expected to be a better starting point for subsequent computational deployment, for example in WScore virtual screening.

In some cases, an improved pose can be obtained via a longer MD simulation. To explore this possibility, we carry out a $100 \mathrm{~ns}$ unbiased MD simulation on the top two poses as ranked by the IFD-MD scoring function of section VI. Regardless of which pose is chosen as the single structure to return to the user, the MD trajectory provides additional information about the structure which can be useful in a variety of applications. We consider the last pose generated in this MD simulation, and refer to it in what follows as the long MD (LMD) pose. 
If the starting PGP pose was sufficiently close to the crystal structure, and there were no issues with the force field or temperature effects, the 100 ns refinement from the PGP pose would be a reliable approach to refining the structure. Note that cases have already been filtered out from the data set based on performance in a $100 \mathrm{~ns}$ MD simulation of the native structure, as stated below in Section XI. However, we have observed some cases in our data set in which the long MD simulation experiences significant drift from the native basin of attraction. In most cases, this is due to distortions of the receptor structure which prevents a collapse into the native basin. In such cases, the BWS pose would be a better choice in typical single structure applications.

Based on the above considerations, we have designed the following simple protocol for selecting the final representative single structure pose for a given PGP pose. We measure the RMSD between the PGP pose and the BWS pose. If the RMSD is less than $2 \AA$, we conclude that the PGP pose is relatively stable, and accept the small (but potentially significant) improvements from the 500 ps MD simulation. If the RMSD is greater than $2 \AA$, we select the LMD. Our empirical observation, based on data from the training set, is that if more extensive relaxation/refinement is required, a $100 \mathrm{~ns}$ MD simulation is more likely (although not guaranteed) to provide satisfactory convergence to a native-like state. The results of this protocol are presented in Section XII.D.

\section{Choosing Between Two or More IFD-MD Poses}

The IFD-MD software has significant synergies with Schrodinger's FEP+ free energy perturbation software ${ }^{13,14}$. Use of relative binding affinity FEP calculations require that experimental binding data for at least one member of a series of congeneric ligands be available. Such information is often available from the experimental literature (e.g. patent filings or a publication by another research group) or can be obtained inexpensively by testing analogues to the target compound available from compound suppliers ("analogue by catalog"). To test the ability of a given structure to recapitulate the binding data, the FEP+ software is utilized in its standard mode of operation, modeling poses for the analogues via core constrained docking, constructing an FEP+ map of alchemical transformations, and carrying out the relevant calculations on a GPU cluster. In the main text, we examine how effectively such FEP+ simulations discriminate correct from incorrect poses for a number of test cases.

An alternative approach, when experimental binding data is not available, is to employ a variation of absolute binding free energy calculations where the two species being compared are not chemically distinct ligands but are simply two conformations of the same ligand-receptor complex. This will be expanded upon fully in a future publication.

When there is ambiguity as to which IFD-MD pose correctly represents the experimental binding mode of a ligand, FEP+ combined with available structure activity relationship data can be employed to discriminate between the alternatives.

The question of how much effort to put into interrogating different IFD-MD clusters depends upon the degree of difficulty of the induced fit docking problem being addressed. We believe that this flexibility is a strength of the approach, allowing users to decide how much computational effort to commit to a particular investigation, considering not only the degree of difficulty of the problem but also the availably of computation time and the importance assigned to obtaining a solution. For example, if one were working on a GPCR target with highly compelling biology, and in competition with a number of other drug discovery groups, a very high priority might be assigned to obtaining an accurate structure with a high degree of confidence as quickly as possible.

\section{Summary of IFD-MD Workflow}

Figure S2 depicts in graphical form a complete summary of the IFD-MD workflow as outlined above. Job control machinery directs computations of various components of the algorithm to the appropriate type of computational cluster (CPU or GPU). Structural visualization tools from Schrödinger's Maestro software are incorporated into the interface, and can be used to view any specific pose indicated by the user.

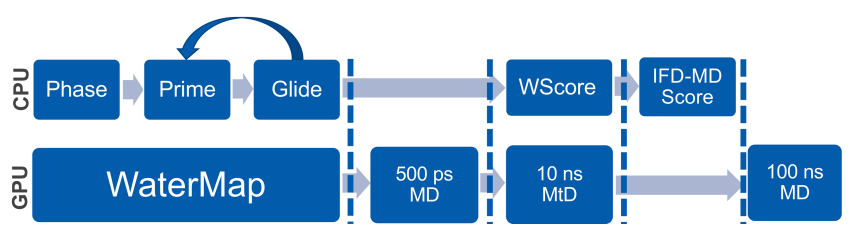

Figure S2. Overview of the IFD-MD Workflow. The horizontal axis indicates time. The blue boxes represent stages. Stages which lie along the same time indicate which steps run in parallel divided across CPU or GPU jobs. The closed loop shown for the Prime/Glide stages 
indicate that these stages iterate once as indicated in Section I.B. Horizontal lines indicate points where the algorithm can be restarted without the need to recompute prior stages. All MD stages, including the Metadynamics (MtD) stage are performed via Desmond.

X Evaluation of IFD-MD Predictions for Use in Free Energy Perturbation Calculations

In a prior publication, we demonstrated the ability to accurately predict relative binding free energies of congeneric ligands for a set of eight diverse systems ${ }^{14,15}$. Here, we look to reproduce these calculations using a ligand-receptor complex originating not from a crystal structure, but from an IFD-MD prediction.

This retrospective study mimics the common scenario where 1) a ligand series exists with affinity data from a biophysical assay 2) no cocrystallized structure for any ligand in the series bound to the target of interest exists 3 ) crystal structures do exist for the identical protein bound to a ligand outside the series. In such a scenario, accurate prediction of the retrospective affinity data from the modelled structures suggest the potential for prospective prediction on that model.

This requires assembling a new set of cross-docks separate from the training or test set. The criteria used for selection are: 1) The template holostructure contain a ligand not present in the congeneric series used for the FEP+ predictions, 2) the resolution of the template crystal structure be better than $2.5 \AA$, and 3) the active site be free of any mutations.

After selection of template structures, at least one ligand from the congeneric series for that system must be docked in with IFD-MD. Assuming limited computational resources, we restrict ourselves to docking in only a single ligand from the entire series. A heuristic is then needed to help decide which congeneric ligand is most likely to produce a predicted ligandreceptor complex competent for all other series ligands. For this purpose, we select the largest ligand in the congeneric series to dock in. However, an alternative strategy would be to dock the most potent ligand instead.

The top two ranked predictions from each IFD-MD job are used for separate FEP+ calculations. By evaluating more than one IFD-MD prediction, we are able to observe the effect of IFD-MD prediction quality on FEP+ calculations. For each IFD-MD prediction, the predicted ligand is used as a reference for automated ligand alignment for the remaining ligands in the congeneric series. Each job is run for 5 ns (per lambda window) using OPLS3e in the 19-3 version of Schrodinger's $\mathrm{FEP}+{ }^{12,16}$.

\section{Data Set Curation Details}

Not all combinations of PDB structures for a single target are suitable for cross-docking. A number of pairs are excluded because of an inconsistent composition of matter. For example, some Protein Kinase A structures with small molecules bound also contain a 20 residue peptide inhibitor (PKI) $)^{17,18}$. Two such structures are PDB IDs 1 SVG and $2 E R G$. This $20-$ residue peptide lies just above the binding site which at a minimum restricts the conformational freedom of the bound ligand. Therefore, 1SVG and 2ERG are valid PDBs for cross-docking but cannot be used to predict crystal structures of ligands for which the PDB cognate receptor structure lacks PKI.

Additionally, cross-dock pairs are excluded when the native structure contains a crystal contact interacting with or near the ligand. Figure S3 shows an example where a crystal mate makes numerous hydrophobic contacts with a saturated ring on the ligand.

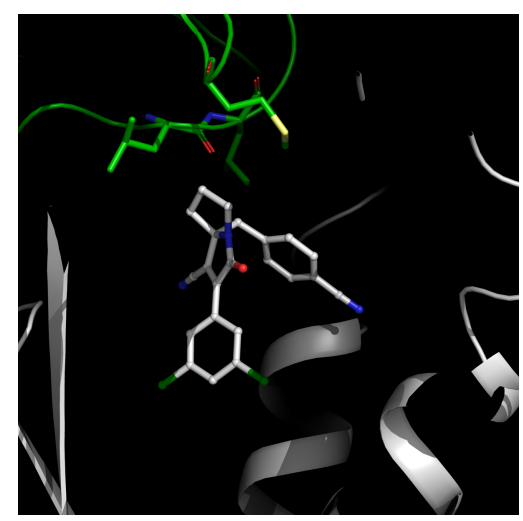

Figure S3. Strong crystal mate interactions between a bound antagonist and crystal mate in lymphocyte function-associated antigen1 (LFA1) structure PDB ID 2O7N. Shown in white is the receptor and ligand in the asymmetric unit. In green is a crystal mate with residues LEU203, LEU204 and MET140 hydrophobically packing against the ligand's saturated ring. 
These structures are still considered valid for template complexes. Cases such as these offer an opportunity to challenge the method when the template ligand contain artifactual effects and are important in confirming the method's low sensitivity to the subtleties of the template ligand.

Finally, we evaluate the suitability of the target structure by running $100 \mathrm{~ns}$ of MD simulation starting from the crystal structure. Ligands which had an average RMSD to the crystal structure of greater than $3.0 \AA$ for the last ns of the simulation are not used as target structures. These cases are still used as template structure, as before, to challenge the method when the starting structure may contain artifactual issues. This MD evaluation here is to interrogate the suitability of the desired crystal structure using an explicit solvent model and the full treatment of our force field. There are a number of reasons why a bound ligand may not retain its binding mode over $100 \mathrm{~ns}$ of MD: uncaught crystal packing effects, temperature effects (comparing the $100 \mathrm{~K}$ temperature using during most $\mathrm{x}$-ray crystallography data collection versus the typical $300 \mathrm{~K}$ MD simulation temperature), as well as possible inaccuracies with our force field or solvent model. These structures are therefore removed from our set of cases in order to isolate failures due solely to our induced fit algorithm rather than the aforementioned possibilities.

\section{XI.A Comparative Methods}

For comparison, IFD-MD is evaluated against our previous IFD release. For comparison against rigid receptor docking, we use GlideSP. Both technologies were evaluated with the Schrodinger 19-3 release ${ }^{19}$.

\section{Detailed Performance of the Individual Stages Within IFD-MD}

XII.A Performance of the Initial Docking Stage.

The first performance metric that we investigate is the best RMSD pose emerging from the Phase-Prime-Glide (PGP) initial docking stage. As discussed previously in Section I.A the ranking of poses at this stage is too crude to be useful, and so instead we report the lowest RMSD complex generated at this stage, regardless of ranking. Figure S4 presents a histogram plot of the number training set cases counted for various values of the lowest observed RMSD.

Previous versions of the complete IFD algorithm fail to produce any low RMSD poses in a nontrivial fraction of test cases ( $7 \%$ when run on the same training set cases that we use here). Performance is satisfactory to within $2.5 \AA$ for all cases across the training set except for two cases $(0.8 \%)$. Of these two cases, one, a PIM1 cross-dock, is refined successfully via molecular dynamics simulation as part of the complete IFD-MD workflow. The second case, a PPAR cross-dock remains a failure.

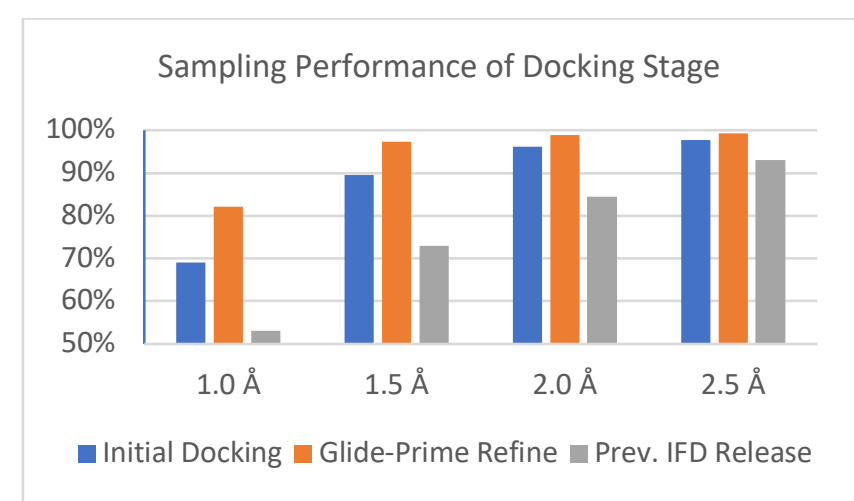

Figure S4. Percentage of training set cases where there exists a predicted ligand receptor complex with a ligand heavy atom RMSD less than or equal to the values shown. The total number of cases is 258 . Initial docking refers to the results immediately after the Phase-GlidePrime initial docking stage. The Glide-Prime refinement is an additional round of GlideSP docking following by Prime receptor refinement that occurs after initial docking. Shown in green is the performance using the previous release of Schrödinger's IFD. This plots the sampling performance of the entire previous IFD algorithm, not any one stage.

XII.B Performance of the Glide-Prime refinement stages 
This stage seeks to accomplish two things: 1) Permit further rounds of side chain optimization and ligand placement. 2) Obtain a more accurate pairing of the Glide docking terms and the Prime energy terms.

The success in achieving the former goal can be seen in Figure S4 which shows the improvement in the minimum RMSD observed compared to the preceding initial docking stage. As was previously stated, these low RMSD poses may in fact be ranked very poorly and so their utility remains undefined until application of an accurate scoring function. This leads to the latter goal.

Figure S5 presents the minimum RMSD seen in the top twenty poses, top ten poses, and top two poses ranked using the preliminary Prime/GlideSP scoring function and incorporating clustering into the ranking as described in Section II. This function is a weighted linear combination of the Prime Energy, GlideSP docking score, and GlideSP Ecoul term. The large drop in the number of successful cases from exploration of the top twenty poses down to the top two poses demonstrates a need for an improved scoring function. Attempts to simply add additional rounds of Glide/Prime calculations does not significantly affect the results presented here.

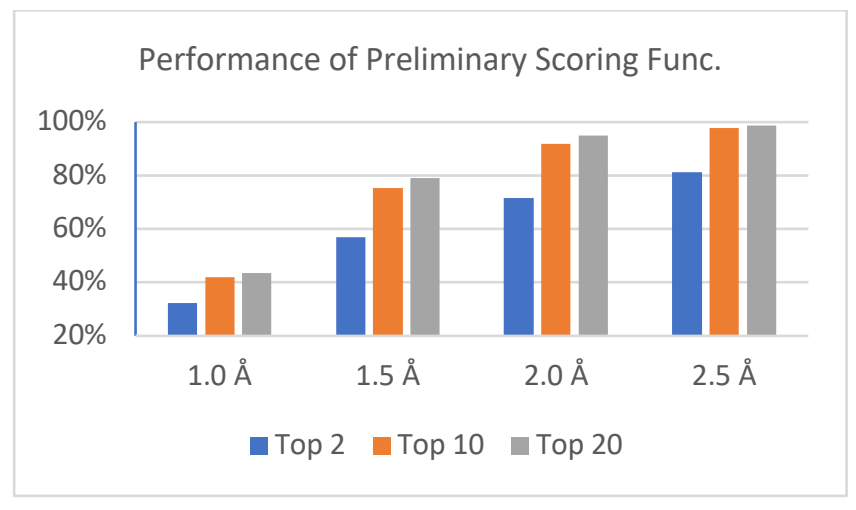

Figure S5. Percentage of cases within the training set where a pose with an RMSD less than the listed threshold was found within the top ranked poses. Shown are the results when exploring the top two poses, top ten poses, and top twenty poses. The training set consists of 258 cross-dock cases.

The percentage of cases in Figure S5 are in general smaller than in Figure S4, as a result of the inability of the preliminary scoring function to carry out perfect rank ordering. However, at least one "native-like" structure (and typically more than one such structure) is present in the top 20 structures in $99 \%$ of cases (failure in three cross-dock cases), and in $98 \%$ of cases in the top 10 structures.

For use in prospective work, we view it as highly desirable to obtain a native-like pose, suitable for FEP+ calculations, within the top two poses. For particularly high value target structures, one can consider extending the search to additional poses (and the capability to do this is available in the IFD-MD framework).

XII.C Optimization and Performance of the Full IFD-MD Scoring Function

The 18 parameters of the full IFD-MD model are optimized by fitting a function of the form given in Equation 1 to the training set data. The objective function to be optimized is defined as

$$
\text { ObjFunc }=-1 * n_{\text {rank } 1 \text { Cases }}-0.2 * n_{\text {rank } 2 \text { Cases }}+0.1 \frac{1}{1+\text { gap }}
$$

Equation 10. Objective Function Used for Parameter Optimization

Here, $n_{\text {rank } 1 \text { Cases }}$ are the number of cases where the top ranked pose had a $2.5 \AA$ ligand heavy atom RMSD or better to the crystal structure, although see below for more detail about how the RMSD was defined, taking into account solvent exposed tails. $n_{\text {rank2 cases }}$ is where the second ranked pose, but not the first, had a $2.5 \AA$ RMSD or better to the crystal structure, and where gap is the score gap between the best ranked pose with an RMSD $2.5 \AA$ or better and the first pose worse than $2.5 \AA$. This gap term only applies to cases where optimization succeeds in ranking a $2.5 \AA$ or better pose within the top two poses. Parameter values resulting from the optimization are given in Table S4. 
Qualitatively, the objective function sought to rank as many quality poses as possible, but still rewards cases where the best quality pose is ranked $2^{\text {nd }}$, as opposed to first. The gap term seeks to optimize the scoring differential between a quality pose and a poor pose. Larger gaps on average imply greater stability of the model when confronted with new cases.

As was previously discussed in section VI, the determination of what constitutes an acceptable pose is non-trivial, particularly when a poor RMSD may be dominated by what appears to be a solvent exposed tail. To quantitatively account for this possibility, the crystal structure is examined over the course of a $100 \mathrm{~ns}$ molecular dynamics simulation. We compare the predicted ligand pose against the native trajectory to see if the native ligand adopts a similar conformation within a quantitative definition as described below. The objective here is to understand whether or not the predicted ligand pose is highly represented in the native MD basin. To distinguish a high degree of representation from transient incidental similarity, we require that at least $25 \%$ of the native MD trajectory lie within $1.5 \AA$ of the predicted ligand pose. When this is found to be the case, the RMSD reported for the prediction is not to the crystal structure but the lowest RMSD against the MD trajectory.

This is consequential in only five of the 258 cases in the training set and one case in the test where the case would have been considered a failure (RMSD $>2.5 \AA$ ) if not for this analysis. Table S1 lists the cases with their RMSDs before and after comparison with native MD. Figure S6 shows the most dramatic case in terms of RMSD change.

Table S1. Cross-docks Considered a Success Due to Comparison with the Native Molecular Dynamics Trajectory

\begin{tabular}{|l|l|l|l|l|}
\hline Target & $\begin{array}{c}\text { Receptor } \\
\text { PDB ID }\end{array}$ & $\begin{array}{c}\text { Ligand } \\
\text { PDB ID }\end{array}$ & $\begin{array}{c}\text { Ligand } \\
\text { Crystal } \\
\text { Structure } \\
\text { RMSD }(\AA)\end{array}$ & $\begin{array}{c}\text { Ligand Crystal } \\
\text { Structure MD } \\
\text { RMSD }(\AA)\end{array}$ \\
\hline CHK1 & $2 \mathrm{C} 3 \mathrm{~K}$ & $2 \mathrm{E} 9 \mathrm{~N}$ & 2.64 & 0.60 \\
\hline CHK1 & $2 \mathrm{C} 3 \mathrm{~L}$ & $2 \mathrm{HXL}$ & 3.67 & 0.59 \\
\hline CHK1 & $2 \mathrm{BRB}$ & $2 \mathrm{E} 9 \mathrm{~N}$ & 3.08 & 0.86 \\
\hline FXA & $1 \mathrm{G} 2 \mathrm{M}$ & $1 \mathrm{FJS}$ & 2.57 & 1.38 \\
\hline FXA & $1 \mathrm{NFX}$ & $1 \mathrm{G} 2 \mathrm{~L}$ & 2.75 & 0.87 \\
\hline LFA1 & $3 \mathrm{BQN}$ & $3 \mathrm{E} 2 \mathrm{M}$ & 2.56 & 0.90 \\
\hline
\end{tabular}
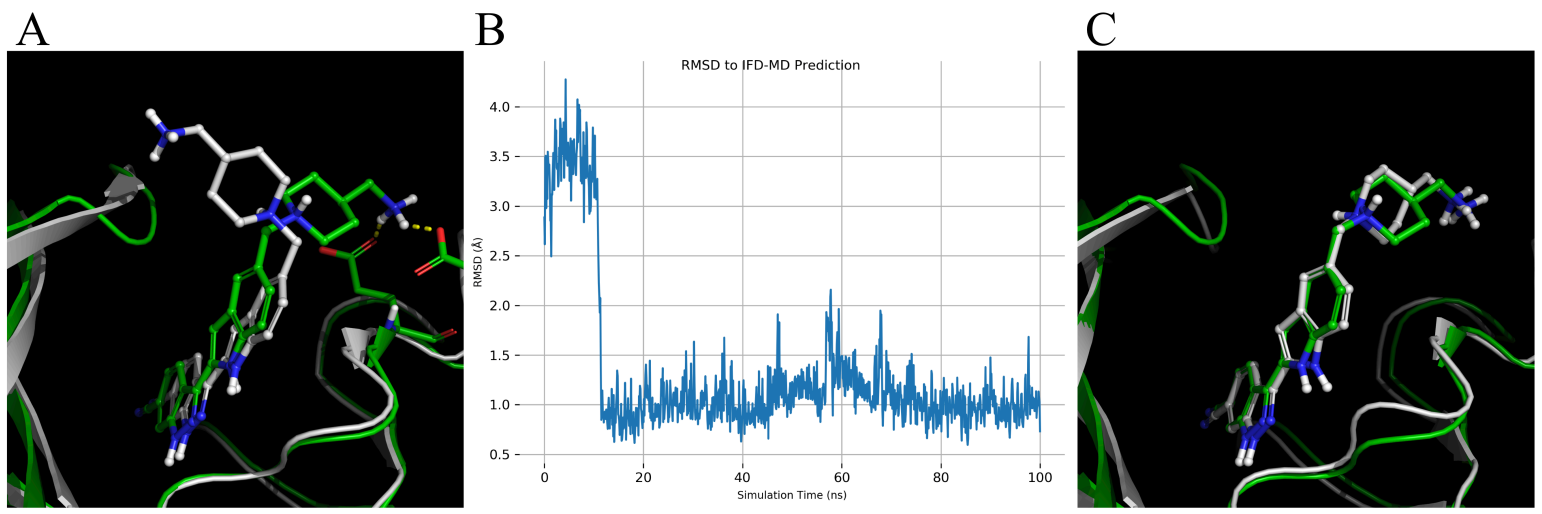

Figure S6. Comparison of the IFD-MD prediction for a CHK1 cross-dock against a molecular dynamics simulation of the native crystal structure. The starting receptor is from PDB ID 2C3L. The ligand being docked in and also the definition of the native structure comes from PDB ID 2HXL. (A). Comparison of the IFD-MD prediction (green) against the crystal structure (white). The ligand heavy atom RMSD is $3.67 \AA$, due almost entirely to the solvent exposed aminomethyl-piperidin tail. IFD-MD prefers to place the positively charged amine in a bridging salt-bridge between GLU92 and ASP95 while the crystal structure points $180^{\circ}$ in the opposite direction. (B) Plot of ligand RMSD versus simulation time for a $100 \mathrm{~ns}$ molecular dynamics simulation of the native crystal structure, 2HXL. The RMSD is computed against the IFD-MD prediction. After approximately $15 \mathrm{~ns}$, the crystal structure ligand collapses into the same basin as the IFDMD prediction. (C) Comparison of the IFD-MD prediction (green) against the native MD frame with the lowest RMSD to the IFD-MD prediction. This occurs at $85.5 \mathrm{~ns}$ and yields an RMSD of 0.59 A. Not shown is the formation of the same bridging salt-bridge as (A). 
Inspection of a $100 \mathrm{~ns}$ molecular dynamics simulation, starting from the crystal structure, indicates that the crystal structure ligand appears to fall into the same basin as the IFD-MD prediction, forming the same bridging salt-bridge as proposed by IFD-MD (Figure S6c). This transition happens at approximately $15 \mathrm{~ns}$ and shows no reversion to the original crystal structure position. Given that the discrepancy between the MD results and the crystal structure may well be due to temperature effects (as noted previously), we count this and other cases as a success for the IFD-MD calculations.

In addition to fitting the entire training set, we also carried out leave one out (LOO) validation by refitting parameters without one arbitrary case, then using the refitted model to rank order the IFD-MD poses for that excluded case. This is done sequentially for each of the 258 cases in the training set. The result for each case is then based on its result when it is excluded from parameter optimization.

Table S2 presents the success rate of the LOO optimized scoring function in ranking the training set cases as either the top pose or as one of the top two poses. Additionally, we show the success rates as each of the major terms in the scoring function is excluded from the model. The significance of a term can be assessed by the drop in the percent of cases which succeeded to within $2.5 \AA$ ligand heavy atom RMSD when that term is removed. Note that we expect the impact of removing multiple terms would be more drastic. While there is physical complementarity between a number of terms below, there are also cases which can succeed due to multiple signals, so that the removal of one term is compensated for by a number of others.

Table S2. Leave-One-Out Optimization Results for the Eight Sub-Functions of the Complete Scoring Function

\begin{tabular}{|l|l|l|}
\hline & \multicolumn{2}{|l|}{ Percent Cases 2.5 $\AA$ RMSD or better } \\
\hline Sub-Function Removed & Top 1 Pose & Top 2 Poses \\
\hline None & $84.9 \%$ & $94.2 \%$ \\
\hline Prime & $77.1 \%$ & $90.3 \%$ \\
\hline GlideSP & $82.9 \%$ & $93.8 \%$ \\
\hline WScore & $77.1 \%$ & $91.5 \%$ \\
\hline PoseStability & $74.0 \%$ & $90.7 \%$ \\
\hline SCMotion & $81.4 \%$ & $92.6 \%$ \\
\hline TemplateOverlap & $81.0 \%$ & $92.2 \%$ \\
\hline
\end{tabular}

The most significant sub-functions are the PoseStability, Prime, and WScore sub-functions, as evidenced by the largest degradation in results occurring when these functions are removed. However, for all sub-functions, the results degrade only marginally (less than $4 \%$ in all cases) if one inspects the output of the top two predictions. These results clearly indicate that many of the cases can be selected via multiple signals. However, if one is interested in the maximum degree of robustness, they also indicate that every term makes an essential contribution to some subset of cases. We view the presence of some degree of overdetermination in the model as helpful in deciding to commit significant resources to progressing a project on the basis of an IFD determined structure. It also will provide some protection against the increased noise that can be expected in more challenging cases, such as those involving loop motion, or in homology modeling. 


\section{A}

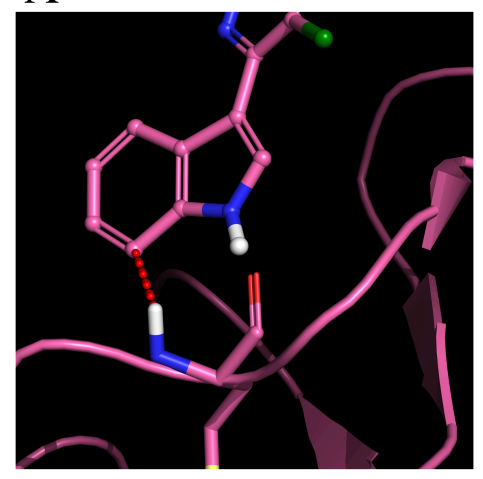

$\mathrm{B}$

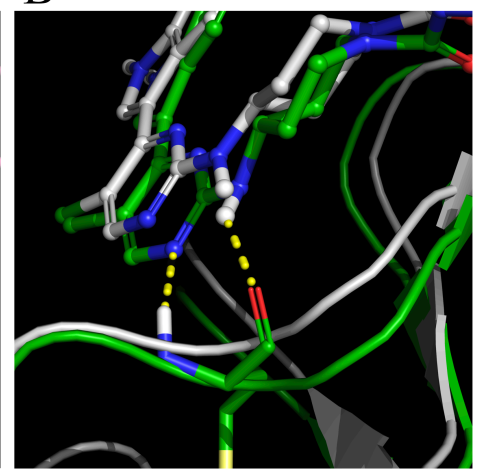

Figure S7. Comparison of predicted poses for a JNK cross-dock highlighting the effect of WScore desolvation terms. The ligand originates from PDB ID 2P33 and the receptor from PDB 1PMV. (A). An 8.0 ^ RMSD prediction. The ligand is forming a single hydrogen bond to the backbone carbonyl of hinge residue MET106. However, the NH on MET106 is desolvated by an aromatic carbon on the indole (shown by the red dotted line). WScore recognizes this desolvation and applies a large penalty to this non-native pose. (B) A 1.170 $\AA$ RMSD prediction in green with the native crystal structure shown in white. In the native-like pose, the proper hinge interaction to MET106 forms using the pyrimidine and adjacent ligand $\mathrm{NH}$.

Although most terms of the scoring function can be difficult to visualize, there are a handful of terms which are straightforward to illustrate. Figure S7 highlights one such example. In this instance, a native-like pose should form a pair of hydrogen bonds to the backbone atoms of MET106 using the pyrimidine and adjacent ligand NH. MET106 forms part of the hinge region. The correct interaction is present in the $1.170 \AA$ pose shown in Figure S7B. The non-native pose forms an incorrect interaction with MET106 using the ligand's indole. This indole can only favorably interact with the MET106 carbonyl; the backbone NH of MET106 is instead desolvated. This desolvation is detected by WScore which returns both a penalty and a lower score. Both of these penalties factor in to the scoring function and result in the non-native pose being ranked $2^{\text {nd }}$ while the native-like pose is ranked $1^{\text {st }}$.

A second term which is easy to visualize is the side chain penalty term. Recall from Section $V$ that this term penalizes the motion of large or heavily interacting side chains relative to their position in the template receptor. This term does not forbid such motion, indeed that may be the correct induced fit effect, but if it's possible to produce an alternative pose without such motion, then with all other terms equal, that pose should be preferred.

Figure S8 highlights an example observed in an HSP90 cross-dock. Here, the native position of PHE131 does not change from the starting receptor, PDB ID 2H55, to the native receptor, PDB ID 1UY6. The structure shown in Figure S8A shows a high RMSD ( $8 \AA$ ligand heavy atom to crystal structure) prediction which has forced the displacement of PHE131 by the placement of hydrophobic aliphatic chains on the ligand. This displacement is not resolved in any of the ten $500 \mathrm{ps}$ MD trials and so this motion receives a large penalty. Figure S8B shows the top-ranked prediction which is a $0.87 \AA$ RMSD pose. PHE131 is correctly placed here with a $0.85 \AA$ heavy-atom side chain RMSD.

These results validate the physical picture described above in which the various IFD-MD scoring components are complementary. Each component addresses one or more physical effects that are difficult to robustly model with the other components. New terms may in fact become necessary as a wider range of test cases is examined; however, the present test set does span a considerable range of targets from the PDB, and we believe that the current implementation is likely to work well in a high percentage of prospective applications. 

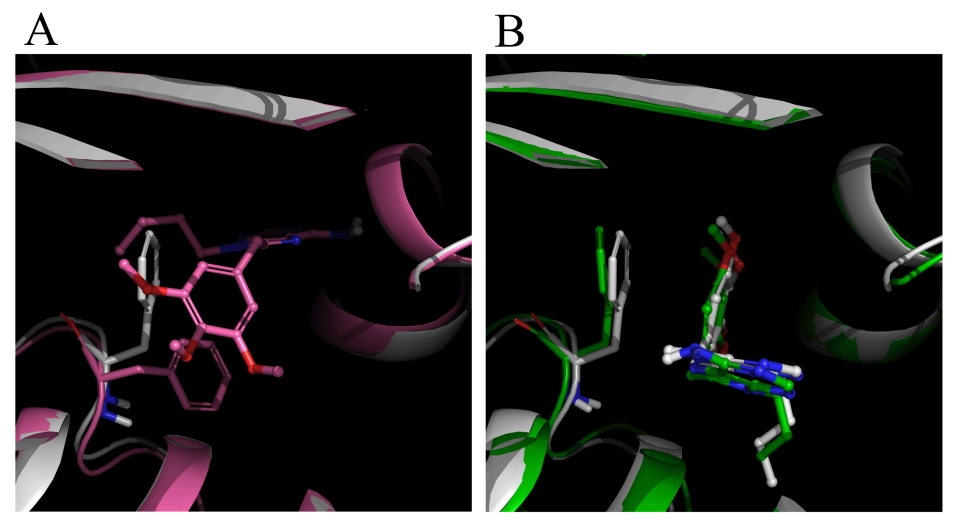

Figure S8. Comparison of predicted poses for an HSP90 cross-dock highlighting the effect of the side chain motion penalty term. The ligand originates from PDB ID 1UY6 and the receptor from PDB 2H55. (A) An $8.2 \AA$ RMSD prediction is shown in pink with the position of PHE131 in the background. The native position of PHE131 is shown in white. PHE131 does not move between the template receptor and the native receptor. The displacement of PHE131, due to the position of the ligand's aliphatic chains, receives a large penalty since it is present in all ten 500 ps MD trials. (B). A $0.87 \AA$ RMSD prediction is shown in green with the native structure shown in white. PHE131 is shown in nearly the exact same position as in the native and has a $0.85 \AA$ side-chain heavy-atom RMSD.

\section{XII.D Performance of the Refinement Stage}

Figure S9 displays a histogram plot showing the distribution of impacts on the first and second ranked IFD-MD poses, from the combined training and test sets, which had RMSDs of $2.5 \AA$ to the native structure either before (the PGP pose) or after (either the BWS pose or the LMD pose, chosen as described in Section VII) refinement. A total of 606 poses in all are included in this evaluation.

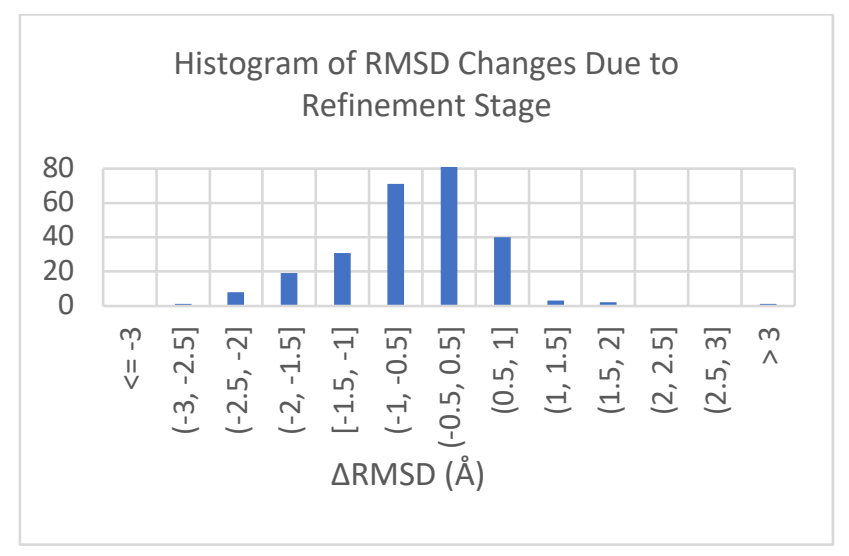

Figure S9. Histogram of ligand heavy-atom RMSD changes, relative to the crystal structure, after the refinement stage when applied to the top two ranked poses. All top two poses within the training and test set are included. Cases which fail to generate a sub $2.5 \AA$ pose, either before or after refinement are excluded. This leaves 606 poses plotted here. To improve visualization, the scale of the y-axis is capped at 80 while the $(-0.5,0.5]$ bin contains a count of 430 poses. The distribution is not symmetric showing that when a pose is altered, it has an overwhelmingly greater tendency towards reducing the RMSD to the crystal structure.

Most of these poses (71\%) do not change much upon refinement (between $0.5 \AA$ and $-0.5 \AA$ change in the RMSD to the native structure pose). However, of the poses that do change significantly, the results are overwhelming biased in a favorable direction. There are on the order of 30 large improvements (greater than $1.5 \AA$ ) as compared to only two significant degradations. These results are consistent across both the training and test sets. The refinement does have limitations; high RMSD poses generally do not collapse to the native structure in $100 \mathrm{~ns}$. Nevertheless, there are a nontrivial number of significant improvements, and the smaller modifications inherent in for example a BWS pose as compared to a PGP pose may prove to be quite useful in subsequent applications. 
Induced Fit Effects. J. Med. Chem. 2006, 49 (2), 534-553. https://doi.org/10.1021/jm050540c.

(2) Zhao, Y.; Sanner, M. F. FLIPDock: Docking Flexible Ligands into Flexible Receptors. Proteins Struct. Funct. Bioinforma. 2007, 68 (3), 726-737. https://doi.org/10.1002/prot.21423.

(3) Bolia, A.; Ozkan, S. B. Adaptive BP-Dock: An Induced Fit Docking Approach for Full Receptor Flexibility. J. Chem. Inf. Model. 2016, 56 (4), 734-746. https://doi.org/10.1021/acs.jcim.5b00587.

(4) Bansal, N.; Zheng, Z.; Merz, K. M. Incorporation of Side Chain Flexibility into Protein Binding Pockets Using MTflex. Bioorg. Med. Chem. 2016, 24 (20), 4978-4987. https://doi.org/10.1016/J.BMC.2016.08.030.

(5) Jacobson, M. P.; Kaminski, G. A.; Friesner, R. A.; Rapp, C. S. Force Field Validation Using Protein Side Chain Prediction. J. Phys. Chem. B 2002, 106 (44), 11673-11680. https://doi.org/10.1021/jp021564n.

(6) Zhu, K.; Shirts, M. R.; Friesner, R. A. Improved Methods for Side Chain and Loop Predictions via the Protein Local Optimization Program: Variable Dielectric Model for Implicitly Improving the Treatment of Polarization Effects. $J$. Chem. Theory Comput. 2007, 3 (6), 2108-2119. https://doi.org/10.1021/ct700166f.

(7) Jacobson, M. P.; Pincus, D. L.; Rapp, C. S.; Day, T. J. F.; Honig, B.; Shaw, D. E.; Friesner, R. A. A Hierarchical Approach to All-Atom Protein Loop Prediction. Proteins Struct. Funct. Bioinforma. 2004, 55 (2), 351-367. https://doi.org/10.1002/prot.10613.

(8) Li, J.; Abel, R.; Zhu, K.; Cao, Y.; Zhao, S.; Friesner, R. A. The VSGB 2.0 Model: A next Generation Energy Model for High Resolution Protein Structure Modeling. Proteins Struct. Funct. Bioinforma. 2011, 79 (10), 2794-2812. https://doi.org/10.1002/prot.23106.

(9) Abel, R.; Young, T.; Farid, R.; Berne, B. J.; Friesner, R. A. Role of the Active-Site Solvent in the Thermodynamics of Factor Xa Ligand Binding. J. Am. Chem. Soc. 2008, 130 (9), 2817-2831. https://doi.org/10.1021/ja0771033.

(10) Murphy, R. B.; Repasky, M. P.; Greenwood, J. R.; Tubert-Brohman, I.; Jerome, S.; Annabhimoju, R.; Boyles, N. A.; Schmitz, C. D.; Abel, R.; Farid, R.; Friesner, R. A. WScore: A Flexible and Accurate Treatment of Explicit Water Molecules in Ligand-Receptor Docking. J. Med. Chem. 2016, 59 (9), 4364-4384. https://doi.org/10.1021/acs.jmedchem.6b00131.

(11) Clark, A. J.; Tiwary, P.; Borrelli, K.; Feng, S.; Miller, E. B.; Abel, R.; Friesner, R. A.; Berne, B. J. Prediction of ProteinLigand Binding Poses via a Combination of Induced Fit Docking and Metadynamics Simulations. J. Chem. Theory Comput. 2016, 12 (6), 2990-2998. https://doi.org/10.1021/acs.jctc.6b00201.

(12) Roos, K.; Wu, C.; Damm, W.; Reboul, M.; Stevenson, J. M.; Lu, C.; Dahlgren, M. K.; Mondal, S.; Chen, W.; Wang, L.; Abel, R.; Friesner, R. A.; Harder, E. D. OPLS3e: Extending Force Field Coverage for Drug-Like Small Molecules. J. Chem. Theory Comput. 2019, 15 (3), 1863-1874. https://doi.org/10.1021/acs.jctc.8b01026.

(13) Abel, R.; Wang, L.; Harder, E. D.; Berne, B. J.; Friesner, R. A. Advancing Drug Discovery through Enhanced Free Energy Calculations. Acc. Chem. Res. 2017, 50 (7), 1625-1632. https://doi.org/10.1021/acs.accounts.7b00083.

(14) Wang, L.; Wu, Y.; Deng, Y.; Kim, B.; Pierce, L.; Krilov, G.; Lupyan, D.; Robinson, S.; Dahlgren, M. K.; Greenwood, J.; Romero, D. L.; Masse, C.; Knight, J. L.; Steinbrecher, T.; Beuming, T.; Damm, W.; Harder, E.; Sherman, W.; Brewer, M.; Wester, R.; Murcko, M.; Frye, L.; Farid, R.; Lin, T.; Mobley, D. L.; Jorgensen, W. L.; Berne, B. J.; Friesner, R. A.; Abel, R. Accurate and Reliable Prediction of Relative Ligand Binding Potency in Prospective Drug Discovery by Way of a Modern Free-Energy Calculation Protocol and Force Field. J. Am. Chem. Soc. 2015, 137 (7), 2695-2703. https://doi.org/10.1021/ja512751q.

(15) Harder, E.; Damm, W.; Maple, J.; Wu, C.; Reboul, M.; Xiang, J. Y.; Wang, L.; Lupyan, D.; Dahlgren, M. K.; Knight, J. L.; Kaus, J. W.; Cerutti, D. S.; Krilov, G.; Jorgensen, W. L.; Abel, R.; Friesner, R. A. OPLS3: A Force Field Providing Broad Coverage of Drug-like Small Molecules and Proteins. J. Chem. Theory Comput. 2016, 12 (1), 281-296. https://doi.org/10.1021/acs.jctc.5b00864.

(16) Schrödinger Release 2020-3: FEP+. Schrodinger, LLC: New York, NY 2020.

(17) Jacobs, M.; Hayakawa, K.; Swenson, L.; Bellon, S.; Fleming, M.; Taslimi, P.; Doran, J. The Structure of Dimeric ROCK I Reveals the Mechanism for Ligand Selectivity. J. Biol. Chem. 2006, 281 (1), 260-268. https://doi.org/10.1074/jbc.M508847200.

(18) Breitenlechner, C. B.; Wegge, T.; Berillon, L.; Graul, K.; Marzenell, K.; Friebe, W.-G.; Thomas, U.; Schumacher, R.; Huber, R.; Engh, R. A.; Masjost, B. Structure-Based Optimization of Novel Azepane Derivatives as PKB Inhibitors. $J$. Med. Chem. 2004, 47 (6), 1375-1390. https://doi.org/10.1021/jm0310479.

(19) Schrödinger Release 2019-3. Schrödinger, LLC: New York, NY 2019.

Table S3. Composition of the training set by receptor and ligand PDBID

\begin{tabular}{|l|l|l|}
\hline Target & Receptor PDBID & Ligand PDBID \\
\hline $\mathrm{a} 2 \mathrm{a}$ & $3 \mathrm{pwh}$ & $3 \mathrm{uza}$ \\
\hline & $3 \mathrm{pwh}$ & $3 \mathrm{uzc}$ \\
\hline & $3 \mathrm{uza}$ & $3 \mathrm{pwh}$ \\
\hline
\end{tabular}




\begin{tabular}{|c|c|c|}
\hline afab & $4 \mathrm{nns}$ & $3 \mathrm{fr} 4$ \\
\hline & $4 \mathrm{nns}$ & $3 \mathrm{fr} 5$ \\
\hline \multirow[t]{2}{*}{ akt1 } & $3 \mathrm{qkk}$ & $3 \mathrm{qkm}$ \\
\hline & $3 \mathrm{qkl}$ & $3 \mathrm{qkm}$ \\
\hline \multirow[t]{16}{*}{ alr2 } & 1iei & $1 z 3 n$ \\
\hline & 1iei & $2 \mathrm{fz} 8$ \\
\hline & 1iei & $2 \mathrm{ikj}$ \\
\hline & $1 \mathrm{pwl}$ & $1 z 3 n$ \\
\hline & 1pwl & $2 \mathrm{hv5}$ \\
\hline & 1pwl & $2 \mathrm{ikg}$ \\
\hline & $1 z 3 n$ & $2 \mathrm{i} 16$ \\
\hline & $2 \mathrm{duz}$ & $2 \mathrm{i} 17$ \\
\hline & $2 \mathrm{fzd}$ & $1 z 89$ \\
\hline & $2 \mathrm{fzd}$ & 1iei \\
\hline & $2 \mathrm{fzd}$ & $1 \mathrm{t} 40$ \\
\hline & $2 \mathrm{fzd}$ & $1 z 3 n$ \\
\hline & $2 \mathrm{fzd}$ & $2 \mathrm{hvn}$ \\
\hline & $2 \mathrm{fzd}$ & 2ikg \\
\hline & $2 \mathrm{i} 17$ & $1 \mathrm{t} 40$ \\
\hline & $2 \mathrm{ikh}$ & $2 \mathrm{fz} 9$ \\
\hline \multirow[t]{3}{*}{ ask1 } & 4bhn & 4bic \\
\hline & 4bhn & 4bid \\
\hline & 4bhn & 4bie \\
\hline \multirow[t]{8}{*}{ bacel } & $4 \mathrm{j} 0 \mathrm{p}$ & $4 \mathrm{n} 00$ \\
\hline & $4 \mathrm{j} 0 \mathrm{p}$ & $4 \mathrm{pzw}$ \\
\hline & 4zsm & $4 \mathrm{zsq}$ \\
\hline & $5 \mathrm{clm}$ & $5 \mathrm{ezx}$ \\
\hline & $5 \mathrm{ezx}$ & $5 \mathrm{f} 00$ \\
\hline & $5 \mathrm{ezx}$ & $5 \mathrm{f} 01$ \\
\hline & $5 \mathrm{f} 00$ & $5 \mathrm{ezx}$ \\
\hline & $5 \mathrm{f01}$ & $5 \mathrm{ezx}$ \\
\hline \multirow[t]{7}{*}{ cdk2 } & $1 \mathrm{~g} 5 \mathrm{~s}$ & $2 \mathrm{~b} 55$ \\
\hline & $1 \mathrm{ke} 5$ & $2 \mathrm{fvd}$ \\
\hline & $1 \mathrm{pxi}$ & $1 \mathrm{e} 1 \mathrm{v}$ \\
\hline & $1 \mathrm{pxj}$ & $1 \mathrm{ke} 8$ \\
\hline & $1 p x j$ & ljvp \\
\hline & $1 p x j$ & $2 \mathrm{a} 0 \mathrm{c}$ \\
\hline & $1 \mathrm{pxj}$ & $2 \mathrm{btr}$ \\
\hline
\end{tabular}




\begin{tabular}{|c|c|c|}
\hline & $1 \mathrm{pxj}$ & $2 \mathrm{bts}$ \\
\hline & $1 \mathrm{pxj}$ & $2 \mathrm{c} 6 \mathrm{~m}$ \\
\hline & $1 \mathrm{pxm}$ & $2 \mathrm{a} 0 \mathrm{c}$ \\
\hline & $1 \mathrm{wcc}$ & 1oit \\
\hline & $1 \mathrm{wcc}$ & $2 \mathrm{~b} 52$ \\
\hline & $1 \mathrm{wcc}$ & $2 \mathrm{bhe}$ \\
\hline & $1 \mathrm{wcc}$ & $2 \mathrm{bts}$ \\
\hline & $1 \mathrm{wcc}$ & $2 \mathrm{c} 6 \mathrm{k}$ \\
\hline & $2 \mathrm{a} 0 \mathrm{c}$ & $1 \mathrm{y} 8 \mathrm{y}$ \\
\hline & $2 \mathrm{btr}$ & $2 \mathrm{c} 6 \mathrm{i}$ \\
\hline & $2 \mathrm{c} 5 \mathrm{y}$ & 1oit \\
\hline & $2 \mathrm{c} 68$ & $1 \mathrm{e} 1 \mathrm{v}$ \\
\hline & $2 \mathrm{c} 6 \mathrm{i}$ & 1pxl \\
\hline & $2 \mathrm{c} 6 \mathrm{i}$ & $1 w 0 x$ \\
\hline \multirow[t]{14}{*}{ chk1 } & $2 \mathrm{brb}$ & $2 \mathrm{hxl}$ \\
\hline & $2 \mathrm{c} 3 \mathrm{j}$ & 1nvs \\
\hline & $2 \mathrm{c} 3 \mathrm{j}$ & $2 \operatorname{cgu}$ \\
\hline & $2 \mathrm{c} 3 \mathrm{j}$ & $2 \mathrm{cgw}$ \\
\hline & $2 \mathrm{c} 3 \mathrm{j}$ & 2 hy 0 \\
\hline & $2 \mathrm{c} 3 \mathrm{k}$ & $2 \mathrm{e} 9 \mathrm{n}$ \\
\hline & $2 \mathrm{c} 31$ & 1nvs \\
\hline & $2 \mathrm{c} 31$ & $2 \mathrm{hxl}$ \\
\hline & $2 \mathrm{e} 9 \mathrm{o}$ & $2 \mathrm{cgu}$ \\
\hline & $2 \mathrm{hog}$ & $2 \operatorname{cgu}$ \\
\hline & $2 \mathrm{hxl}$ & $2 \mathrm{brn}$ \\
\hline & $2 \mathrm{hxq}$ & 2ayp \\
\hline & $2 \mathrm{hxq}$ & $2 \operatorname{cgu}$ \\
\hline & 2hy0 & $2 \mathrm{brb}$ \\
\hline \multirow[t]{3}{*}{ dhodh } & $3 \mathrm{kvk}$ & $3 \mathrm{kvl}$ \\
\hline & 4jgd & $3 \mathrm{kvl}$ \\
\hline & 4jgd & $3 u 2 o$ \\
\hline \multirow[t]{7}{*}{ dpp4 } & $1 \mathrm{n} 1 \mathrm{~m}$ & $2 \mathrm{fjp}$ \\
\hline & $\ln 1 \mathrm{~m}$ & $1 \times 70$ \\
\hline & $\ln 1 \mathrm{~m}$ & 2aj8 \\
\hline & $1 \mathrm{n} 1 \mathrm{~m}$ & 2oph \\
\hline & 1rwq & $2 \mathrm{fjp}$ \\
\hline & 1rwq & $1 \times 70$ \\
\hline & 1rwq & 2aj8 \\
\hline
\end{tabular}




\begin{tabular}{|c|c|c|}
\hline & 1rwq & $2 o p h$ \\
\hline & 2iiv & $1 \mathrm{n} 1 \mathrm{~m}$ \\
\hline \multirow[t]{9}{*}{ er } & $1 \mathrm{u} 3 \mathrm{q}$ & $1 \times 76$ \\
\hline & $1 \mathrm{u} 3 \mathrm{r}$ & $1 \times 76$ \\
\hline & $1 \mathrm{u} 3 \mathrm{r}$ & $1 \times 78$ \\
\hline & $1 \mathrm{u} 3 \mathrm{r}$ & $1 \times 7 b$ \\
\hline & $1 \mathrm{u} 3 \mathrm{~s}$ & $1 \mathrm{u} 3 \mathrm{r}$ \\
\hline & $1 \times 78$ & $1 \mathrm{u} 3 \mathrm{r}$ \\
\hline & 1yy4 & $1 \mathrm{u} 3 \mathrm{~s}$ \\
\hline & 1yye & $1 \mathrm{u} 3 \mathrm{r}$ \\
\hline & $2 \mathrm{i} 0 \mathrm{~g}$ & $1 \mathrm{u} 3 \mathrm{~s}$ \\
\hline \multirow[t]{6}{*}{ fviia } & 1wqv & $1 w t g$ \\
\hline & 1wqv & $2 \mathrm{flb}$ \\
\hline & lyge & 1wss \\
\hline & 1ygc & $1 \mathrm{z} 6 \mathrm{j}$ \\
\hline & $2 \mathrm{a} 2 \mathrm{q}$ & 1wss \\
\hline & $2 \mathrm{flb}$ & 1wss \\
\hline \multirow[t]{21}{*}{ fxa } & $1 \mathrm{~g} 2 \mathrm{~m}$ & $1 \mathrm{fjs}$ \\
\hline & $1 \mathrm{~g} 2 \mathrm{~m}$ & $11 \mathrm{pk}$ \\
\hline & $1 \mathrm{~g} 2 \mathrm{~m}$ & $2 b q 7$ \\
\hline & liqm & $2 \mathrm{boh}$ \\
\hline & 1lqd & 1iqm \\
\hline & $1 \mathrm{mq} 6$ & lioe \\
\hline & $1 \mathrm{mq} 6$ & $1 \mathrm{ksn}$ \\
\hline & $1 \mathrm{nfx}$ & 1g21 \\
\hline & $\operatorname{lnfx}$ & $11 p g$ \\
\hline & $1 \mathrm{nfx}$ & $11 \mathrm{pk}$ \\
\hline & $1 \mathrm{nfx}$ & $11 \mathrm{pz}$ \\
\hline & $1 \mathrm{nfx}$ & $11 q d$ \\
\hline & $1 x k a$ & 1lpk \\
\hline & $1 \mathrm{xka}$ & $11 p z$ \\
\hline & $1 \mathrm{xka}$ & 1lqd \\
\hline & $1 x k a$ & $1 \mathrm{mq} 5$ \\
\hline & 1xka & $2 \mathrm{bq} 6$ \\
\hline & 1xka & $2 \mathrm{bq} 7$ \\
\hline & $2 \mathrm{bmg}$ & $2 \mathrm{bq} 6$ \\
\hline & 2bok & $2 \mathrm{bqw}$ \\
\hline & $2 \mathrm{cji}$ & $2 b q 7$ \\
\hline
\end{tabular}




\begin{tabular}{|c|c|c|}
\hline \multirow[t]{3}{*}{ glur5 } & 3fuz & $3 \mathrm{fv} 2$ \\
\hline & 3fuz & $3 \mathrm{fvg}$ \\
\hline & $3 \mathrm{fv} 1$ & 3 fuz \\
\hline \multirow[t]{2}{*}{ gsk3b } & 4acd & $4 \mathrm{acc}$ \\
\hline & 4ach & $4 \mathrm{acc}$ \\
\hline \multirow[t]{9}{*}{ hivrt } & $1 \mathrm{klm}$ & $1 \mathrm{vrt}$ \\
\hline & $1 \mathrm{vrt}$ & 1vru \\
\hline & $2 \mathrm{~b} 5 \mathrm{j}$ & $1 \mathrm{dtq}$ \\
\hline & $2 \mathrm{~b} 5 \mathrm{j}$ & $1 \mathrm{c} 0 \mathrm{u}$ \\
\hline & $2 \mathrm{~b} 5 \mathrm{j}$ & 1 eet \\
\hline & $2 \mathrm{~b} 5 \mathrm{j}$ & $1 \mathrm{fkp}$ \\
\hline & $2 \mathrm{~b} 5 \mathrm{j}$ & $1 \mathrm{rt6}$ \\
\hline & $2 \mathrm{~b} 5 \mathrm{j}$ & $1 \mathrm{~s} 1 \mathrm{t}$ \\
\hline & $2 \mathrm{~b} 5 \mathrm{j}$ & $1 \mathrm{tkx}$ \\
\hline \multirow[t]{3}{*}{ hsd1 } & 2irw & $3 \operatorname{ch} 6$ \\
\hline & $4 i j u$ & $4 i j v$ \\
\hline & $4 \mathrm{ijv}$ & 4iju \\
\hline \multirow[t]{3}{*}{ hsp70 } & $3 \mathrm{fzh}$ & $3 \mathrm{~m} 3 \mathrm{z}$ \\
\hline & $3 \mathrm{fzl}$ & $3 \mathrm{~m} 3 \mathrm{z}$ \\
\hline & $31 \mathrm{dq}$ & $3 \mathrm{~m} 3 \mathrm{z}$ \\
\hline \multirow[t]{6}{*}{ hsp90 } & 1yet & 2byi \\
\hline & $2 \mathrm{bsm}$ & $2 \mathrm{ccu}$ \\
\hline & 2 byh & 2byi \\
\hline & $2 \mathrm{cdd}$ & $2 \mathrm{ccu}$ \\
\hline & $2 \mathrm{fwz}$ & 1uy6 \\
\hline & $2 \mathrm{~h} 55$ & 1uy6 \\
\hline $\operatorname{irak} 4$ & $4 \mathrm{ztm}$ & 4yp8 \\
\hline \multirow[t]{3}{*}{ jnk } & $1 \mathrm{pmv}$ & $2 \mathrm{p} 33$ \\
\hline & $2 \mathrm{o} 0 \mathrm{u}$ & $1 \mathrm{pmn}$ \\
\hline & $2 \mathrm{o} 2 \mathrm{u}$ & $1 \mathrm{pmv}$ \\
\hline \multirow[t]{3}{*}{ lck } & 1qpe & 2of4 \\
\hline & $2 o f u$ & 2of2 \\
\hline & $2 o f u$ & 2of4 \\
\hline \multirow[t]{5}{*}{ Ifa1 } & $1 \mathrm{xdg}$ & $3 \mathrm{bqn}$ \\
\hline & $2 \mathrm{ica}$ & 3bqn \\
\hline & $207 n$ & 1 cqp \\
\hline & $3 \mathrm{bqm}$ & $3 \mathrm{e} 2 \mathrm{~m}$ \\
\hline & 3bqn & $3 \mathrm{e} 2 \mathrm{~m}$ \\
\hline
\end{tabular}




\begin{tabular}{|c|c|c|}
\hline & $3 \mathrm{e} 2 \mathrm{~m}$ & $1 \mathrm{cqp}$ \\
\hline \multirow[t]{3}{*}{$\mathrm{mdm} 2$} & $3 \mathrm{jzk}$ & $4 z y f$ \\
\hline & $3 w 69$ & 4jv9 \\
\hline & 4hg7 & $4 z y f$ \\
\hline \multirow[t]{2}{*}{$\mathrm{mk} 2$} & $2 \mathrm{p} 3 \mathrm{~g}$ & 3 fyj \\
\hline & $3 \mathrm{~m} 2 \mathrm{w}$ & $3 \mathrm{kga}$ \\
\hline \multirow[t]{5}{*}{ mps 1} & $3 \mathrm{gfw}$ & 5ehy \\
\hline & $3 \mathrm{hmo}$ & $4 c 4 i$ \\
\hline & $4 c 4 f$ & 5ehy \\
\hline & $4 c 4 i$ & 5ehy \\
\hline & $4 c 4 j$ & 5 ei6 \\
\hline \multirow[t]{7}{*}{ oppa } & $1 \mathrm{~b} 05$ & $1 \mathrm{~b} 0 \mathrm{~h}$ \\
\hline & 1b0h & $1 b 31$ \\
\hline & $1 \mathrm{~b} 31$ & $1 \mathrm{~b} 0 \mathrm{~h}$ \\
\hline & 1b31 & $1 \mathrm{~b} 3 \mathrm{~h}$ \\
\hline & $1 \mathrm{~b} 46$ & $1 \mathrm{~b} 0 \mathrm{~h}$ \\
\hline & $1 b 52$ & $1 \mathrm{~b} 0 \mathrm{~h}$ \\
\hline & $1 \mathrm{jet}$ & $1 \mathrm{~b} 0 \mathrm{~h}$ \\
\hline \multirow[t]{5}{*}{ p38 } & $1 \mathrm{bl} 7$ & $1 \mathrm{w} 84$ \\
\hline & $1 \mathrm{w} 7 \mathrm{~h}$ & 1уqj \\
\hline & $1 \mathrm{wbv}$ & $1 w b n$ \\
\hline & $1 w b v$ & $1 \mathrm{wbs}$ \\
\hline & $2 \mathrm{baj}$ & $1 w 82$ \\
\hline \multirow[t]{5}{*}{ pde10a } & $3 w s 8$ & 5edi \\
\hline & 3ws9 & 3 ws 8 \\
\hline & $4 \mathrm{fcb}$ & 4 phw \\
\hline & 4heu & $4 \mathrm{p} 1 \mathrm{r}$ \\
\hline & $4 p h w$ & 3 ws8 \\
\hline \multirow[t]{5}{*}{ pde5 } & $2 \mathrm{chm}$ & $1 \times p 0$ \\
\hline & 3 sie & $2 \mathrm{~h} 44$ \\
\hline & $4 \mathrm{md} 6$ & 1uho \\
\hline & $4 \mathrm{md} 6$ & 3 shy \\
\hline & 4oew & 3 shy \\
\hline \multirow[t]{5}{*}{$\mathrm{pdk}$} & 1uu3 & $3 \mathrm{sc} 1$ \\
\hline & 1uu9 & 1uu8 \\
\hline & 1uu9 & 3rwp \\
\hline & 3nun & $3 \mathrm{sc} 1$ \\
\hline & $3 \mathrm{scl}$ & 3nun \\
\hline
\end{tabular}




\begin{tabular}{|c|c|c|}
\hline \multirow[t]{7}{*}{ pim1 } & $2 x i z$ & $41 \mathrm{mu}$ \\
\hline & $3 \mathrm{vbt}$ & $41 \mathrm{mu}$ \\
\hline & $3 \mathrm{vc} 4$ & $3 b g q$ \\
\hline & $4 \mathrm{bzn}$ & $3 \mathrm{vbq}$ \\
\hline & $4 \mathrm{i} 41$ & 3umw \\
\hline & $4 \operatorname{lm} 5$ & $3 \mathrm{vbx}$ \\
\hline & $41 \mathrm{mu}$ & 4bzo \\
\hline \multirow[t]{5}{*}{ pka } & 1re8 & 1rek \\
\hline & 1rej & $1 \mathrm{bx} 6$ \\
\hline & $1 \mathrm{xh} 4$ & $1 \times 46$ \\
\hline & $2 \mathrm{c} 1 \mathrm{a}$ & $1 \mathrm{bx} 6$ \\
\hline & 2erz & $1 \mathrm{svg}$ \\
\hline \multirow[t]{13}{*}{ ppar } & 1nyx & $2 \mathrm{~g} 0 \mathrm{~g}$ \\
\hline & $2 \mathrm{fvj}$ & $2 \mathrm{~g} 0 \mathrm{~h}$ \\
\hline & $2 \mathrm{fvj}$ & 1nyx \\
\hline & $2 \mathrm{fvj}$ & 2ath \\
\hline & $2 \mathrm{fvj}$ & $2 \mathrm{~g} 0 \mathrm{~g}$ \\
\hline & $2 \mathrm{i} 4 \mathrm{z}$ & $1 \mathrm{i} 7 \mathrm{i}$ \\
\hline & $2 \mathrm{i} 4 \mathrm{z}$ & $2 \mathrm{~g} 0 \mathrm{~g}$ \\
\hline & 2 prg & $2 \mathrm{f} 4 \mathrm{~b}$ \\
\hline & $2 \mathrm{prg}$ & $2 \mathrm{i} 4 \mathrm{j}$ \\
\hline & $2 \mathrm{prg}$ & 2ath \\
\hline & $2 \mathrm{prg}$ & $2 \mathrm{~g} 0 \mathrm{~g}$ \\
\hline & 2 prg & 2gtk \\
\hline & 2 prg & 1zgy \\
\hline \multirow[t]{6}{*}{ ptp1b } & $1 \mathrm{c} 87$ & $2 \mathrm{~h} 4 \mathrm{~g}$ \\
\hline & $1 \mathrm{c} 88$ & $2 \mathrm{~b} 07$ \\
\hline & $1 \mathrm{ecv}$ & $2 \mathrm{hb} 1$ \\
\hline & 1wax & $1 \mathrm{c} 84$ \\
\hline & $2 \mathrm{bgd}$ & $2 \mathrm{~cm} 7$ \\
\hline & $2 \mathrm{~cm} 8$ & $2 \mathrm{~h} 4 \mathrm{k}$ \\
\hline rho & $2 \mathrm{esm}$ & 2etk \\
\hline \multirow[t]{2}{*}{ rpa } & 4lwc & $4 \mathrm{r} 4 \mathrm{o}$ \\
\hline & $4 \mathrm{r} 4 \mathrm{c}$ & 4lwc \\
\hline \multirow[t]{4}{*}{ throm } & $1 \mathrm{a} 4 \mathrm{w}$ & $1 \mathrm{t} 4 \mathrm{u}$ \\
\hline & 1ae8 & $1 \mathrm{~d} 9 \mathrm{i}$ \\
\hline & 1ae8 & 1gj4 \\
\hline & 1ae8 & 1gj5 \\
\hline
\end{tabular}




\begin{tabular}{|l|l|l|}
\hline & $1 \mathrm{ae} 8$ & $1 \mathrm{o} 2 \mathrm{~g}$ \\
\hline & $1 \mathrm{ae} 8$ & $1 \mathrm{o} 5 \mathrm{~g}$ \\
\hline & $1 \mathrm{c} 5 \mathrm{o}$ & $1 \mathrm{nzq}$ \\
\hline & $1 \mathrm{~d} 4 \mathrm{p}$ & $1 \mathrm{~d} 9 \mathrm{i}$ \\
\hline & $1 \mathrm{gj} 5$ & $1 \mathrm{~d} 3 \mathrm{~d}$ \\
\hline & $1 \mathrm{jwt}$ & $1 \mathrm{~d} 9 \mathrm{i}$ \\
\hline & $1 \mathrm{jwt}$ & $1 \mathrm{tom}$ \\
\hline & $1 \mathrm{k} 21$ & $1 \mathrm{a} 4 \mathrm{w}$ \\
\hline & $1 \mathrm{k} 21$ & $1 \mathrm{~d} 3 \mathrm{~d}$ \\
\hline & $1 \mathrm{k} 21$ & $1 \mathrm{riw}$ \\
\hline & $1 \mathrm{~nm} 6$ & $1 \mathrm{~d} 9 \mathrm{i}$ \\
\hline & $1 \mathrm{~nm} 6$ & $1 \mathrm{fpc}$ \\
\hline & $1 \mathrm{nzq}$ & $1 \mathrm{sl3}$ \\
\hline & $1 \mathrm{qbv}$ & $1 \mathrm{a} 4 \mathrm{w}$ \\
\hline & $1 \mathrm{ta} 2$ & $1 \mathrm{o} 0 \mathrm{~d}$ \\
\hline tryp & $2 \mathrm{bm} 2$ & $3 \mathrm{v} 7 \mathrm{t}$ \\
\hline & $2 \mathrm{za} 5$ & $3 \mathrm{v} 7 \mathrm{t}$ \\
\hline & $3 \mathrm{v} 7 \mathrm{t}$ & $5 \mathrm{f03}$ \\
\hline & $4 \mathrm{a} 61$ & $3 \mathrm{v} 7 \mathrm{t}$ \\
\hline & $5 \mathrm{f} 03$ & $2 \mathrm{za} 5$ \\
\hline & $5 \mathrm{f03}$ & $3 \mathrm{v} 7 \mathrm{t}$ \\
\hline & $1 \mathrm{c5w}$ & $1 \mathrm{gj} 9$ \\
\hline & $1 \mathrm{c5y}$ & $1 \mathrm{gj} 9$ \\
\hline & $1 \mathrm{gjc}$ & $1 \mathrm{sc} 8$ \\
\hline & $1 \mathrm{sc} 8$ & $1 \mathrm{o3p}$ \\
\hline & $2 \mathrm{vio}$ & $1 \mathrm{c5w}$ \\
\hline & $1 \mathrm{w} 10$ & $1 \mathrm{f} 92$ \\
\hline & & \\
\hline & & \\
\hline & & \\
\hline & &
\end{tabular}


Table S4. Composition of the test set by receptor and ligand PDBID

\begin{tabular}{|c|c|c|}
\hline Target & Receptor PDBID & Ligand PDBID \\
\hline afab & $2 n n q$ & $3 \mathrm{fr} 4$ \\
\hline \multirow[t]{5}{*}{ alr2 } & $1 \mathrm{pwm}$ & 2ine \\
\hline & $1 \mathrm{pwm}$ & 2 inz \\
\hline & $1 z 89$ & $1 \mathrm{t} 40$ \\
\hline & $2 \mathrm{ikh}$ & 2 inz \\
\hline & $2 n v c$ & 2 inz \\
\hline \multirow[t]{3}{*}{ bace1 } & $4 \mathrm{j} 0 \mathrm{p}$ & $5 \mathrm{ezx}$ \\
\hline & $4 z s p$ & $4 \mathrm{zsq}$ \\
\hline & $4 \mathrm{zsq}$ & $5 \mathrm{hd} 0$ \\
\hline \multirow[t]{20}{*}{ cdk2 } & $1 \mathrm{~h} 1 \mathrm{q}$ & $\log u$ \\
\hline & 1jsv & $1 \mathrm{pxk}$ \\
\hline & ljsv & 1vyz \\
\hline & $1 \mathrm{jsv}$ & $1 \mathrm{y} 8 \mathrm{y}$ \\
\hline & $1 \mathrm{jsv}$ & $2 \mathrm{a} 0 \mathrm{c}$ \\
\hline & ljsv & $2 \mathrm{btr}$ \\
\hline & $1 \mathrm{pxi}$ & $1 \mathrm{jsv}$ \\
\hline & $1 \mathrm{pxi}$ & $1 y 8 y$ \\
\hline & 1pxi & $2 \mathrm{c} 6 \mathrm{k}$ \\
\hline & $1 \mathrm{pxj}$ & $1 \mathrm{jsv}$ \\
\hline & $1 \mathrm{pxj}$ & $1 \mathrm{pxl}$ \\
\hline & $1 \mathrm{pxj}$ & $1 \mathrm{y} 8 \mathrm{y}$ \\
\hline & $1 \mathrm{pxj}$ & $2 \mathrm{c} 6 \mathrm{k}$ \\
\hline & $1 \mathrm{pxj}$ & $2 \mathrm{c} 61$ \\
\hline & $2 \mathrm{a} 0 \mathrm{c}$ & $1 p x p$ \\
\hline & $2 \mathrm{a} 0 \mathrm{c}$ & $2 \mathrm{c} 61$ \\
\hline & $2 c 69$ & $1 \mathrm{e} 1 \mathrm{v}$ \\
\hline & $2 \mathrm{c} 6 \mathrm{i}$ & $1 \mathrm{pxk}$ \\
\hline & $2 \mathrm{c} 6 \mathrm{i}$ & $1 \mathrm{pxp}$ \\
\hline & $2 \mathrm{c} 61$ & $1 \mathrm{pxk}$ \\
\hline \multirow[t]{6}{*}{ chk1 } & lnvs & $2 \mathrm{hog}$ \\
\hline & $2 \mathrm{brb}$ & $2 e 9 n$ \\
\hline & $2 \mathrm{brg}$ & $2 \mathrm{e} 9 \mathrm{n}$ \\
\hline & $2 \mathrm{c} 3 \mathrm{k}$ & $2 \operatorname{cgu}$ \\
\hline & $2 \mathrm{c} 3 \mathrm{k}$ & $2 \operatorname{cg} x$ \\
\hline & $2 \mathrm{cgu}$ & 2 ayp \\
\hline
\end{tabular}




\begin{tabular}{|c|c|c|}
\hline & $2 \operatorname{cgu}$ & $2 \mathrm{c} 3 \mathrm{k}$ \\
\hline \multirow[t]{10}{*}{ dpp4 } & $\ln 1 \mathrm{~m}$ & $2 \mathrm{hha}$ \\
\hline & $1 \mathrm{n} 1 \mathrm{~m}$ & $2 \mathrm{iiv}$ \\
\hline & 1rwq & 2iiv \\
\hline & $1 \times 70$ & $2 \mathrm{fjp}$ \\
\hline & $2 \mathrm{fjp}$ & 1rwq \\
\hline & $2 \mathrm{fjp}$ & $2 \mathrm{hha}$ \\
\hline & $2 \operatorname{og} z$ & $1 \times 70$ \\
\hline & $2 \operatorname{og} z$ & $2 \mathrm{fjp}$ \\
\hline & $2 o p h$ & $1 \times 70$ \\
\hline & $2 o p h$ & $2 \mathrm{fjp}$ \\
\hline \multirow[t]{7}{*}{ er } & $1 \mathrm{u} 3 \mathrm{r}$ & 1yye \\
\hline & $1 \mathrm{u} 3 \mathrm{~s}$ & 1yy4 \\
\hline & $1 \mathrm{u} 9 \mathrm{e}$ & $1 y y 4$ \\
\hline & $1 \times 76$ & $1 \mathrm{u} 3 \mathrm{r}$ \\
\hline & $1 \times 7 b$ & $1 \mathrm{u} 3 \mathrm{~s}$ \\
\hline & 1yy4 & $1 u 3 r$ \\
\hline & 1yye & $1 \mathrm{u} 3 \mathrm{~s}$ \\
\hline \multirow[t]{2}{*}{ fviia } & 1wss & 1wun \\
\hline & $1 \mathrm{wv} 7$ & 1wss \\
\hline \multirow[t]{18}{*}{ fxa } & 1 fax & $1 \mathrm{fjs}$ \\
\hline & $1 \mathrm{fjs}$ & 1 fax \\
\hline & $1 \mathrm{fjs}$ & 1wu1 \\
\hline & $1 \mathrm{fjs}$ & $1 x k a$ \\
\hline & $1 \mathrm{fjs}$ & $1 \times k b$ \\
\hline & 1g21 & 1 fax \\
\hline & 1g21 & 1lqd \\
\hline & $1 \mathrm{~g} 21$ & $2 \mathrm{bq} 6$ \\
\hline & $1 \mathrm{~g} 21$ & $2 \mathrm{bq} 7$ \\
\hline & $1 \mathrm{~g} 2 \mathrm{~m}$ & $1 \mathrm{mq} 6$ \\
\hline & $1 \mathrm{ksn}$ & $1 \times k b$ \\
\hline & $1 \mathrm{mq} 5$ & $1 \mathrm{fjs}$ \\
\hline & $1 \mathrm{mq} 6$ & $11 \mathrm{pg}$ \\
\hline & 1wul & $11 \mathrm{pg}$ \\
\hline & 1wu1 & 1lpk \\
\hline & 1wu1 & $11 \mathrm{pz}$ \\
\hline & 1wu1 & $11 q d$ \\
\hline & 1wul & $1 \mathrm{xkb}$ \\
\hline
\end{tabular}




\begin{tabular}{|c|c|c|}
\hline & $1 \mathrm{xka}$ & $1 \mathrm{ksn}$ \\
\hline & $1 \mathrm{xkb}$ & $11 \mathrm{pk}$ \\
\hline & $1 \mathrm{xkb}$ & $1 \mathrm{lpz}$ \\
\hline & $1 \mathrm{xkb}$ & 1lqd \\
\hline & $1 \mathrm{xkb}$ & $2 \mathrm{bq} 7$ \\
\hline glur5 & $3 f u z$ & $3 \mathrm{fvk}$ \\
\hline \multirow[t]{2}{*}{ gsk3b } & $3 \mathrm{sd} 0$ & 4ach \\
\hline & $4 \mathrm{ach}$ & $3 \mathrm{sd} 0$ \\
\hline hsp90 & $2 \mathrm{bt} 0$ & $2 \mathrm{ccu}$ \\
\hline \multirow[t]{6}{*}{ oppa } & $1 \mathrm{~b} 32$ & $1 \mathrm{~b} 0 \mathrm{~h}$ \\
\hline & $1 \mathrm{~b} 3 \mathrm{f}$ & $1 \mathrm{~b} 0 \mathrm{~h}$ \\
\hline & $1 \mathrm{~b} 4 \mathrm{z}$ & $1 \mathrm{~b} 0 \mathrm{~h}$ \\
\hline & $1 \mathrm{~b} 51$ & $1 \mathrm{~b} 0 \mathrm{~h}$ \\
\hline & $1 \mathrm{~b} 5 \mathrm{i}$ & $\mathrm{lb} 0 \mathrm{~h}$ \\
\hline & $1 \mathrm{~b} 5 \mathrm{j}$ & $1 \mathrm{~b} 0 \mathrm{~h}$ \\
\hline \multirow[t]{4}{*}{ pde10a } & 3ws9 & $4 \mathrm{fcb}$ \\
\hline & 3ws9 & 5edi \\
\hline & $4 \mathrm{fcb}$ & 3ws9 \\
\hline & $4 \mathrm{fcb}$ & 5edi \\
\hline $\mathrm{pdk}$ & 3rwp & $3 \mathrm{sc} 1$ \\
\hline \multirow[t]{15}{*}{ pim1 } & 1yi3 & 2064 \\
\hline & 1yi3 & 3r04 \\
\hline & 1yi3 & $4 a 7 c$ \\
\hline & $1 \mathrm{yi} 3$ & $4 \mathrm{bzo}$ \\
\hline & $1 \mathrm{yi} 3$ & $4 \mathrm{enx}$ \\
\hline & 1yi3 & $4 \mathrm{j} \times 7$ \\
\hline & 2064 & $4 \mathrm{enx}$ \\
\hline & $2 x j 2$ & $4 \mathrm{enx}$ \\
\hline & $3 b g q$ & $4 \mathrm{enx}$ \\
\hline & $3 \mathrm{bgz}$ & 4enx \\
\hline & $3 \mathrm{vbx}$ & $203 p$ \\
\hline & $3 \mathrm{vbx}$ & 3uix \\
\hline & $3 \mathrm{vbx}$ & 4 bzn \\
\hline & $3 \mathrm{vbx}$ & $4 \mathrm{bzo}$ \\
\hline & $3 \mathrm{vbx}$ & $4 \operatorname{lm} 5$ \\
\hline \multirow[t]{2}{*}{ ppar } & $2 \mathrm{~g} 0 \mathrm{~g}$ & $2 \mathrm{prg}$ \\
\hline & $2 \mathrm{i} 4 \mathrm{j}$ & $2 \mathrm{~g} 0 \mathrm{~g}$ \\
\hline ptp1b & $1 \mathrm{c} 83$ & $2 \mathrm{~cm} 7$ \\
\hline
\end{tabular}




\begin{tabular}{|c|c|c|}
\hline & $1 \mathrm{c} 84$ & $1 \mathrm{xbo}$ \\
\hline & $1 \mathrm{c} 84$ & $2 \mathrm{~cm} 7$ \\
\hline & $1 \mathrm{c} 84$ & $2 \mathrm{~h} 4 \mathrm{~g}$ \\
\hline & $1 \mathrm{c} 87$ & $2 \mathrm{~cm} 7$ \\
\hline & $1 \mathrm{c} 87$ & $2 \mathrm{~h} 4 \mathrm{k}$ \\
\hline & $1 \mathrm{c} 88$ & $1 \mathrm{xbo}$ \\
\hline & $1 \mathrm{c} 88$ & $2 \mathrm{~cm} 7$ \\
\hline & $1 \mathrm{c} 88$ & $2 \mathrm{~h} 4 \mathrm{~g}$ \\
\hline & $1 \mathrm{c} 88$ & $2 \mathrm{~h} 4 \mathrm{k}$ \\
\hline & 1no6 & 1 pyn \\
\hline & 1wax & $1 \times b o$ \\
\hline & 1wax & $2 \mathrm{~cm} 7$ \\
\hline & 1wax & $2 \mathrm{~h} 4 \mathrm{~g}$ \\
\hline & 1wax & $2 \mathrm{~h} 4 \mathrm{k}$ \\
\hline & 1xbo & $2 \mathrm{~cm} 7$ \\
\hline & $2 \mathrm{~b} 07$ & $1 \times b o$ \\
\hline & $2 \mathrm{~b} 07$ & $2 \mathrm{~cm} 7$ \\
\hline rho & $2 \mathrm{esm}$ & $2 \mathrm{etr}$ \\
\hline rpa & $4 \mathrm{r} 4 \mathrm{o}$ & $4 r 4 t$ \\
\hline \multirow[t]{19}{*}{ throm } & $1 c 5 n$ & $1 d 3 p$ \\
\hline & $1 c 5 n$ & $1 d 4 p$ \\
\hline & $1 \mathrm{c} 5 \mathrm{n}$ & 1gj4 \\
\hline & $1 c 5 n$ & 1gj5 \\
\hline & $1 c 5 n$ & $1 \mathrm{k} 21$ \\
\hline & $1 c 5 n$ & $1 \mathrm{ktt}$ \\
\hline & $1 c 5 n$ & 1nzq \\
\hline & $1 c 5 n$ & $100 d$ \\
\hline & $1 c 5 n$ & $105 \mathrm{~g}$ \\
\hline & $1 \mathrm{~d} 3 \mathrm{p}$ & 1gj4 \\
\hline & $1 \mathrm{~d} 3 \mathrm{p}$ & 1gj5 \\
\hline & $1 \mathrm{~d} 3 \mathrm{p}$ & $105 \mathrm{~g}$ \\
\hline & $1 \mathrm{~d} 9 \mathrm{i}$ & 1gj5 \\
\hline & $1 \mathrm{~d} 9 \mathrm{i}$ & $105 \mathrm{~g}$ \\
\hline & $1 g 32$ & $1 d 4 p$ \\
\hline & $1 \mathrm{~g} 32$ & $105 \mathrm{~g}$ \\
\hline & 1ghv & $105 \mathrm{~g}$ \\
\hline & 1ghw & $105 \mathrm{~g}$ \\
\hline & $1 \mathrm{k} 21$ & $105 \mathrm{~g}$ \\
\hline
\end{tabular}




\begin{tabular}{|l|l|l|}
\hline tryp & $3 \mathrm{v} 7 \mathrm{t}$ & $4 \mathrm{a} 61$ \\
\hline upa & $1 \mathrm{c} 5 \mathrm{w}$ & $1 \mathrm{gj} 7$ \\
\hline & $1 \mathrm{c} 5 \mathrm{w}$ & $1 \mathrm{gjb}$ \\
\hline & $1 \mathrm{c} 5 \mathrm{w}$ & $1 \mathrm{gjc}$ \\
\hline & $1 \mathrm{c} 5 \mathrm{x}$ & $1 \mathrm{gj} 7$ \\
\hline & $1 \mathrm{c} 5 \mathrm{x}$ & $1 \mathrm{gj} 9$ \\
\hline & $1 \mathrm{c} 5 \mathrm{x}$ & $1 \mathrm{gjb}$ \\
\hline uro & $1 \mathrm{f} 51$ & $1 \mathrm{f} 92$ \\
\hline
\end{tabular}


Table S5. Count of cross-docks by target for both the training and test set

\begin{tabular}{|c|c|}
\hline Target & $\begin{array}{l}\text { Number of Cross-docks } \\
\text { (Training/Test Set) }\end{array}$ \\
\hline $\mathrm{A} 2 \mathrm{a}$ & $3 / 0$ \\
\hline Afab & $2 / 1$ \\
\hline Akt1 & $2 / 0$ \\
\hline Alr2 & $16 / 5$ \\
\hline Ask1 & $3 / 0$ \\
\hline Bace1 & $8 / 3$ \\
\hline Cdk2 & $21 / 20$ \\
\hline Chk1 & $14 / 7$ \\
\hline Dhodh & $3 / 0$ \\
\hline Dpp4 & $9 / 10$ \\
\hline $\mathrm{Er}$ & $9 / 7$ \\
\hline Fviia & $6 / 2$ \\
\hline Fxa & $21 / 23$ \\
\hline Glur5 & $3 / 1$ \\
\hline Gsk3b & $2 / 2$ \\
\hline Hivrt & $9 / 0$ \\
\hline Hsd1 & $3 / 0$ \\
\hline Hsp70 & $3 / 0$ \\
\hline Hsp90 & $6 / 1$ \\
\hline Irak4 & $1 / 0$ \\
\hline Jnk & $3 / 0$ \\
\hline Lck & $3 / 0$ \\
\hline Lfa1 & $6 / 0$ \\
\hline $\mathrm{Mdm} 2$ & $3 / 0$ \\
\hline $\mathrm{Mk} 2$ & $2 / 0$ \\
\hline Mps1 & $5 / 0$ \\
\hline Oppa & $7 / 6$ \\
\hline P38 & $5 / 0$ \\
\hline Pde10a & $5 / 4$ \\
\hline Pde5 & $5 / 0$ \\
\hline Pdk & $5 / 1$ \\
\hline Pim1 & $7 / 15$ \\
\hline $\mathrm{Pka}$ & $5 / 0$ \\
\hline Ppar & $13 / 2$ \\
\hline Ptp1b & $6 / 18$ \\
\hline Rho & $1 / 1$ \\
\hline Rpa & $2 / 1$ \\
\hline Throm & $19 / 19$ \\
\hline Tryp & $6 / 1$ \\
\hline
\end{tabular}




\begin{tabular}{|l|l|}
\hline Upa & $5 / 6$ \\
\hline Uro & $1 / 1$ \\
\hline Total & $258 / 157$ \\
\hline
\end{tabular}

Table S6. Parameter values for the composite scoring function

\begin{tabular}{|c|l|}
\hline Parameter & Value \\
\hline$w_{\mathbf{1}}$ & 0.36 \\
\hline$w_{\mathbf{2}}$ & 0.8 \\
\hline$w_{\mathbf{3}}$ & 0.4 \\
\hline$w_{\mathbf{4}}$ & 4.2 \\
\hline$w_{\mathbf{5}}$ & 0.36 \\
\hline$w_{\mathbf{6}}$ & 1.6 \\
\hline$w_{\mathbf{7}}$ & -1.4 \\
\hline$w_{\mathbf{8}}$ & 5 \\
\hline$P_{\mathbf{1}}$ & 0 \\
\hline$P_{\mathbf{2}}$ & 0.6 \\
\hline$w_{\mathbf{9}}$ & 2.28 \\
\hline$w_{\mathbf{1 0}}$ & 0.84 \\
\hline$M_{\text {SCPen,Phe }}$ & 5 \\
\hline$M_{\text {SCPen,Met }}$ & 2 \\
\hline$M_{\text {SCPen,General }}$ & 10 \\
\hline$w_{\mathbf{1 1}}$ & 6 \\
\hline$w_{\mathbf{1 2}}$ & 4.8 \\
\hline$\epsilon$ & 0.17 \\
\hline & \\
\hline & \\
\hline & \\
\hline & \\
\hline & \\
\hline & \\
\hline & \\
\hline
\end{tabular}

Table S7. Composition of the expanded test set by receptor and ligand PDBID

\begin{tabular}{|l|l|l|}
\hline Target & Receptor PDBID & Ligand PDBID \\
\hline ar & $2 \mathrm{hvc}$ & $2 \mathrm{am} 9$ \\
\hline & $2 \mathrm{ax} 6$ & $3 \mathrm{rll}$ \\
\hline pgr & $1 \mathrm{a} 28$ & $1 \mathrm{sr} 7$ \\
\hline & $1 \mathrm{e} 3 \mathrm{k}$ & $2 \mathrm{w} 8 \mathrm{y}$ \\
\hline pde4 & $2 \mathrm{pw} 3$ & $6 \mathrm{f} 6 \mathrm{u}$ \\
\hline jnk1 & $3 \mathrm{pze}$ & $3 \mathrm{elj}$ \\
\hline thrb & $3 \mathrm{jzc}$ & $1 \mathrm{xzx}$ \\
\hline dck & $4 \mathrm{jln}$ & $4 \mathrm{kcg}$ \\
\hline & $4 \mathrm{jln}$ & $4 \mathrm{q} 1 \mathrm{f}$ \\
\hline dhfr & $1 \mathrm{mvs}$ & $1 \mathrm{dhf}$ \\
\hline
\end{tabular}




\begin{tabular}{|l|l|l|}
\hline & $3 \mathrm{nxo}$ & $1 \mathrm{dhf}$ \\
\hline & $3 \mathrm{~s} 9 \mathrm{u}$ & $3 \mathrm{fl} 8$ \\
\hline fabp4 & $3 \mathrm{p} 6 \mathrm{~g}$ & $2 \mathrm{nnq}$ \\
\hline & $5 \mathrm{edc}$ & $2 \mathrm{nnq}$ \\
\hline & $5 \mathrm{edc}$ & $5 \mathrm{~d} 45$ \\
\hline hivpr & $1 \mathrm{hvh}$ & $1 \mathrm{hvi}$ \\
\hline & $3 \mathrm{oxw}$ & $2 \mathrm{r3t}$ \\
\hline pnmt & $3 \mathrm{kqt}$ & 2 nny \\
\hline tyk2 & $4 \mathrm{ytc}$ & 5 tq5 \\
\hline
\end{tabular}

